\title{
Exploitation of Source Nonstationarity in Underdetermined Blind Source Separation With Advanced Clustering Techniques
}

\author{
Yuhui Luo, Member, IEEE, Wenwu Wang, Member, IEEE, Jonathon A. Chambers, Senior Member, IEEE, \\ Sangarapillai Lambotharan, Member, IEEE, and Ian Proudler
}

\begin{abstract}
The problem of blind source separation (BSS) is investigated. Following the assumption that the time-frequency (TF) distributions of the input sources do not overlap, quadratic TF representation is used to exploit the sparsity of the statistically nonstationary sources. However, separation performance is shown to be limited by the selection of a certain threshold in classifying the eigenvectors of the TF matrices drawn from the observation mixtures. Two methods are, therefore, proposed based on recently introduced advanced clustering techniques, namely Gap statistics and self-splitting competitive learning (SSCL), to mitigate the problem of eigenvector classification. The novel integration of these two approaches successfully overcomes the problem of artificial sources induced by insufficient knowledge of the threshold and enables automatic determination of the number of active sources over the observation. The separation performance is thereby greatly improved. Practical consequences of violating the TF orthogonality assumption in the current approach are also studied, which motivates the proposal of a new solution robust to violation of orthogonality. In this new method, the TF plane is partitioned into appropriate blocks and source separation is thereby carried out in a block-by-block manner. Numerical experiments with linear chirp signals and Gaussian minimum shift keying (GMSK) signals are included which support the improved performance of the proposed approaches.
\end{abstract}

Index Terms-Gap statistics, self-splitting competitive learning (SSCL), time-frequency (TF) representation, underdetermined blind source separation (BSS).

\section{INTRODUCTION}

$\mathbf{T}$ HE problem of underdetermined blind source separation (BSS) has received considerable research interest over the last decade due to potential applications in various areas including digital communications, financial time series analysis, and medical imaging [4]-[6]. Given a set of linear mixtures, which is typically picked up by an array of sensors,

Manuscript received September 6, 2004; revised July 8, 2005. This work was supported by QinetiQ under the United Kingdom Ministry of Defence Corporate Research Programme CISP. The associate editor coordinating the review of this manuscript and approving it for publication was Dr. Jonathan H. Manton.

Y. Luo is with Samsung Electronics Research Institute, Communications House, Middlesex, TW18 4QE, U.K. (e-mail: yuhuiluo@samsung.com).

W. Wang is with Tao Group Ltd., Reading, RG6 1AZ, U.K. (e-mail: wenwuw@tao-group.com).

J. A. Chambers and S. Lambotharan are with the Centre of DSP, Cardiff School of Engineering, Cardiff University, Cardiff CF24 OYF, U.K. (e-mail: chambersj@cf.ac.uk; lambotharans@ cardiff.ac.uk).

I. Proudler is with the Advanced Signal Processing Group, QinetiQ, Malvern Technology Centre, Worcs, WR14 3PS, U.K. (e-mail: i.proudler@signal.qinetiq.com).

Digital Object Identifier 10.1109/TSP.2006.873367 the aim of BSS is to extract the underlying source signals from the mixtures without explicit prior knowledge of the transmission channels and original sources. Mathematically, denote $\mathbf{s}=\left[s_{1}, s_{2}, \ldots, s_{k}\right]^{T} \in \mathbb{C}^{k \times 1}$ as the vector of source signals and $\mathbf{x}=\left[x_{1}, x_{2}, \ldots, x_{m}\right]^{T} \in \mathbb{C}^{m \times 1}$ as the received signals from the sensor array. The instantaneous mixing process as a function of discrete time, denoted by $t$, is written as

$$
\mathbf{x}(t)=A \mathbf{s}(t)+\mathbf{n}(t)
$$

where $A=\left[\mathbf{a}_{1} \ldots \mathbf{a}_{k}\right] \in \mathbb{C}^{m \times k}$ is a mixing matrix, the column vector $\mathbf{a}_{i}$ is the steering vector corresponding to the source $s_{i}$ and $\mathbf{n}(t)$ is the possible additive noise. In the standard BSS problem, the following three assumptions are conventionally required.

A1) The input sources $s_{1}(t), \ldots, s_{k}(t)$ are statistically mutually independent.

A2) At most one source has Gaussian distribution.

A3) The mixing matrix $A$ has full column rank.

Assumption A1 is the cornerstone of the so-called approach of independent component analysis (ICA). To restore independence that is lost after linear mixing by (1), a separating or unmixing matrix $W \in \mathbb{C}^{m \times k}$ is introduced at the sensor outputs. The recovered source signals, denoted by $\mathbf{y}(t)=\left[y_{1}(t), y_{2}(t), \ldots, y_{k}(t)\right]^{T}$, can be written as

$$
\mathbf{y}(t)=W \mathbf{x}(t)=W A \mathbf{s}(t) .
$$

Ideally, when $W A=I, \mathbf{y}(t)$ will be a perfect estimation of $\mathbf{s}(t)$, where $I$ represents an identity matrix. Practically, however, there exists inherent scaling and permutation ambiguities in ICA as a consequence of restoring the independence of the sources. That is, the unmixing solution becomes

$$
W A=P \Lambda
$$

where $P$ is a permutation matrix and $\Lambda$ is a diagonal scaling matrix. These ambiguities can be easily explained, as any finite nonzero constant multiplying the sources can be cancelled out by dividing the corresponding column of the mixing matrix by the same constant. At the same time, it is not possible to predetermine the order of the sources when both the sources and the mixing matrix are unknown.

Assumption A2 is a direct consequence of assumption A1, for the sum of any two Gaussian sources can be represented by another Gaussian source with composite coefficients. Assumption 
A3 can be interpreted as a requirement regarding the number of measurement sensors and that the sources are generated from spatially distinct locations. That is, in a multiple-input, multiple-output (MIMO) system, $m \geqslant k$. Many successful approaches have been developed for the standard BSS problem such as the well-known JADE algorithm [1], FastICA [6], Informax algorithm [2] and natural gradient algorithms [3]. For more references, readers are referred to research monographs or review papers such as [4]-[9].

In many practical applications, however, the assumption of $m \geqslant k$ is difficult to satisfy. Noticing the significance of such concern, in this paper, we are particularly interested in the possibility of separating more sources than mixtures $(m<k)$, i.e., the problem of underdetermined (or overcomplete) BSS. Compared with the standard BSS problem, underdetermined BSS is more difficult due to the following two reasons. First, in a $k$ sources and $m$ sensors system $(k>m)$, the rank of the spatial correlation matrix of the received signal $R_{\mathrm{xx}}$ cannot exceed $m$, which leads to difficulty if we wish to apply the conventional approach of whitening and rotation as in standard BSS. Second, even if the mixing matrix is successfully estimated, the estimation of the input sources in underdetermined BSS is not as straightforward as that when the number of sensors is sufficient, since there is no unique inverse of the mixing matrix or the inverse may not even exist.

Most of the available approaches resort to exploiting the temporal sparsity inherent to input sources. For example, in [12]-[16], an overcomplete signal representation (also see [11]), which is essentially based on the assumption that the signals have a sparse distribution such as a Laplacian distribution, is exploited for underdetermined BSS. Signal separation is accomplished by an operation in two steps, i.e., the estimation of the basis vectors and then the estimation of the sources. However, with this method, the estimation of the basis vectors may not be very accurate. Similarly, in [17], the mixing matrix is adaptively estimated with a gradient type algorithm, or the k-means clustering method. The difficulty of there being an infinite number of solutions in inversion is tackled by selecting the sparse solution with minimum 1-norm. For sources that are not sufficiently temporally sparse, the author suggested the usage of wavelet packets so that sparsity in the transform domain can be exploited rather than the time domain. This type of minimum 1-norm estimation of input sources is also suggested in [18].

These approaches attempt to estimate both the mixing matrix and the input sources. In comparison, the focus of some other literature is on the estimation of either the mixing matrix or the input sources, but not both. For example, the FOCUSS algorithm [23] exploits the temporal sparsity of the input sources by assuming that at any time only one source is present. The inverse matrix is estimated as the one that maximizes the sparsity of the unmixing outputs. In the case that this sparsity assumption does not hold, the solution will, however, not be unique. Several improved FOCUSS-based algorithms were designed to solve the underdetermined inverse problem in [24] and [25]. Unfortunately, only a locally optimal solution can be obtained with this approach and also solutions are not unique. Assuming perfect knowledge of the mixing matrix, different probabilistic source sparsity models are suggested in [19] for the identification of the transmitting source. In contrast, methods proposed in [20] and [21] concentrate on the estimation of the mixing matrix by decomposing a set of fourth-order cumulants tensors into rank-1 matrices with an eigenvalue decomposition, based on the rank of a fourth order tensor potentially being much higher than the number of input sources. A frequency domain approach based on similar ideas can be found in [22].

The techniques of TF representation for underdetermined BSS are exploited in [26]-[31]. Most of these approaches assume that the TF representations of input sources do not overlap in the transform domain. In [26]-[28], for example, the orthogonality assumption is exploited to separate an arbitrary number of sources from two mixtures. In [31], however, this assumption is suggested for separating nonstationary signals in a quadratic TF domain. But a major drawback of the suggested algorithm is that it often leads to phantom sources being identified because of the approximate classification technique employed. Thresholding in the later stage therefore becomes crucial and its operation requires the number of active sources to be known a priori. In [37] and [38], advanced clustering approaches have been proposed for active source selection in underdetermined BSS. In this paper, these approaches will be further developed and explored in more detail. We will show for the first time that, by using relatively recent advanced clustering techniques, the aforementioned drawback in [31] can be removed and therefore the separation performance be significantly improved. The situation when the orthogonality assumption of the TF distributions of sources is violated is moreover addressed. A new block-based robust approach is proposed by relaxing the TF orthogonality assumption, [39].

The remainder of this paper is organized as follows. Quadratic TF representation of signals is briefly introduced in Section II. An analysis of the orthogonality assumption of TF distribution of sources required for underdetermined BSS is given in Section III. The downside of an approach exploiting this assumption is also highlighted in this section. In Section IV, we present two new algorithms for active source selection required for performing underdetermined BSS in the sense of TF representation. These algorithms are developed on the basis of two advanced clustering techniques, i.e., the methods using Gap statistics and SSCL. The violation of the orthogonality assumption and the corresponding solutions are detailed in Section V. Finally, the performance of the proposed approaches is examined in Section VI and the conclusions are given in Section VII.

\section{QUADRATIC TF REPRESENTATION AND SignaL NONSTATIONARITY}

TF representation provides a useful signal processing tool for characterizing signals in both time and frequency planes. Depending on whether it satisfies the linear superposition principle, it can be classified as linear or quadratic (bilinear) [36] TF representation. For linear transforms such as the short-time Fourier transform and the wavelet transform, the linear superposition principle holds, i.e., the transform of the linear combination of two signal components $x(t)=c_{1} x_{1}(t)+c_{2} x_{2}(t)$ is equal to the same linear combination of the transform of each component, 
where $c_{i}(i=1,2)$ are constants and $x(t)$ is a multicomponent signal [36], [41]. For a quadratic TF representation such as the Wigner distribution [36], however, the transform of $x(t)$ is given by

$$
\begin{aligned}
T_{x}(t, f)=\left|c_{1}\right|^{2} T_{x_{i}}( & t, f)+\left|c_{2}\right|^{2} T_{x_{j}}(t, f) \\
& +c_{1} c_{2}^{*} T_{x_{i} x_{j}}(t, f)+c_{1}^{*} c_{2} T_{x_{j} x_{i}}(t, f)
\end{aligned}
$$

where $T_{x_{i}}(t, f)$ is the transform of signal component $x_{i}(t)$, the term $T_{x_{i} x_{j}}(t, f)$ is the cross TF representation of the two signal components $x_{i}(t)$ and $x_{j}(t)$, and the notation $(\cdot)^{*}$ denotes the complex conjugate operation. The quadratic distribution provides a natural way of representing the energy (or correlation) of signals, which is also a quadratic signal representation [36].

However, a major issue of quadratic TF representation comes from the interference due to the cross TF term $T_{x_{i} x_{j}}(t, f)$, as the two signal components may be buried among the interference. To improve the concentration of the signal components, various kinds of kernel functions have been introduced to reduce the amount of interference. Among which, we will employ Cohen's class of TF distribution due to its desirable property of TF shift invariance [32]. That is, if the signal is delayed in time and/or shifted in frequency, its TF representation will be shifted by the same time delay and/or modulation. The discrete-time form of Cohen's class of TF representation for vector sensor signals $\mathbf{x}$ is given by [10]

$D_{\mathbf{x x}}(t, f)=\sum_{l=-\infty}^{\infty} \sum_{v=-\infty}^{\infty} \boldsymbol{\phi}(v, l) \mathbf{x}(t+v-l) \mathbf{x}^{H}(t+v+l) e^{-j 4 \pi f l}$

where $\phi(v, l)$ is a matrix whose $(i, j)$ th entry $\phi_{i j}(v, l)$ is the kernel associated with the $i$ th and the $j$ th measurement sensor output and $(\cdot)^{H}$ denotes Hermitian transpose. According to (1), $D_{\mathbf{x x}}(t, f)$ relates to the transform of the input sources, for a noise-free situation, by

$$
D_{\mathbf{x x}}(t, f)=A D_{\mathbf{s s}}(t, f) A^{H}
$$

where

$$
D_{\mathbf{s} \mathbf{s}}(t, f)=\sum_{l=-\infty}^{\infty} \sum_{v=-\infty}^{\infty} \phi(v, l) \mathbf{s}(t+v-l)
$$

$\mathbf{s}^{H}(t+v+l) e^{-j 4 \pi f l}$ is the TF matrix of the input sources.

In this paper, we will highlight the statistical nonstationarity of signals by the exploitation of spatial TF representation. As aforementioned, TF representation provides a powerful tool to exploit the signal nonstationarity for achieving source separation. The statistical nonstationarity of signals makes it possible to identify the TF regions where the underdetermined issue is converted into an exactly or overdetermined case, which is not possible to realize for stationary signals. Therefore, the exploitation of TF representation and signal nonstationarity paves a way for solving the underdetermined BSS problem, as verified by our paper.

\section{EXPLOITING TF ORTHOGONALITY IN UNDERDETERMINED BSS}

To exploit source nonstationarity in underdetermined BSS, a crucial assumption for using the TF representation as in [31], is that the TF supports of different sources, $\Omega_{i}$ and $\Omega_{j}$, for example, are orthogonal (without overlap between each other), i.e. $\Omega_{i} \cap \Omega_{j}=0, i \neq j$, where $i$ and $j$ are source indexes. A direct consequence of this assumption is that, for all time and frequency combinations $(t, f)$, only one diagonal entry of $D_{\mathbf{x x}}(t, f)$ will be different from zero. Hence, if at the point $\left(t_{i}, f_{i}\right)$, only source- $j$ is present, $D_{\mathbf{x x}}\left(t_{i}, f_{i}\right)$ is a rank-1 matrix which can be expressed as

$$
D_{\mathbf{x x}}\left(t_{i}, f_{i}\right)=D_{s_{j} s_{j}}\left(t_{i}, f_{i}\right) \mathbf{a}_{j} \mathbf{a}_{j}^{H}
$$

where $D_{s_{j} s_{j}}\left(t_{i}, f_{i}\right)$ is the TF distribution of source- $j$ at $\left(t_{i}, f_{i}\right)$ and it can be estimated as the principle eigenvalue of $D_{\mathbf{x x}}\left(t_{i}, f_{i}\right)$. The eigen decomposition of $D_{\mathbf{x x}}\left(t_{i}, f_{i}\right)$ is given by

$$
D_{\mathbf{x x}}\left(t_{i}, f_{i}\right)=V\left(t_{i}, f_{i}\right) \Lambda\left(t_{i}, f_{i}\right) V^{H}\left(t_{i}, f_{i}\right)
$$

where $V\left(t_{i}, f_{i}\right)$ is a unitary matrix whose column vectors $\mathbf{v}\left(t_{i}, f_{i}\right)$ are eigenvectors, and $\Lambda\left(t_{i}, f_{i}\right)$ is a diagonal matrix whose diagonal elements are the corresponding eigenvalues. Comparing (7) and (8), it is clear that there is only a phase difference between $\mathbf{v}\left(t_{i}, f_{i}\right)$ and $\mathbf{a}_{j}$, which can be mitigated by a rotation operation to $\mathbf{v}\left(t_{i}, f_{i}\right)$ such that its first element becomes a real scalar [31]. For two different TF points, $\left(t_{1}, f_{1}\right)$ and $\left(t_{2}, f_{2}\right)$, for example, if they are associated with the same source, $s_{j}$, it is straightforward to deduce from (7), that the steering vectors associated with matrices $D_{\mathbf{x x}}\left(t_{1}, f_{1}\right)$ and $D_{\mathbf{x x}}\left(t_{2}, f_{2}\right)$ are actually the same, i.e. $\mathbf{a}_{j}$. This implies that the separation problem can be converted into the problem of grouping the TF points which correspond to the same normalized principal eigenvectors (subject to a phase mitigation). The source signals can then be synthesized with the estimator of $D_{s_{j} s_{j}}(t, f), j=1 \ldots k$, by using suitable source synthesis methods such as [41].

A crucial problem in this approach is the classification of the set of eigenvectors obtained at different TF points. In [31], two normalized vectors $\widetilde{\mathbf{v}}\left(t_{1}, f_{1}\right)$ and $\widetilde{\mathbf{v}}\left(t_{2}, f_{2}\right)$ drawn from two TF points are allocated into different classes if the angle between them is larger than a certain threshold, i.e.,

$$
\arccos \left(\widetilde{\mathbf{v}}^{T}\left(t_{1}, f_{1}\right) \widetilde{\mathbf{v}}\left(t_{2}, f_{2}\right)\right)>\varepsilon
$$

where $\widetilde{\mathbf{v}}\left(t_{i}, f_{i}\right)=\left[\operatorname{Re}\left(\mathbf{v}\left(t_{i}, f_{i}\right)\right)^{T} \operatorname{Im}\left(\mathbf{v}\left(t_{i}, f_{i}\right)\right)^{T}\right]^{T}$ and its Euclidean norm $\left\|\widetilde{\mathbf{v}}\left(t_{i}, f_{i}\right)\right\|_{2}=1$. However, there is no available guideline to select $\varepsilon$. As indicated by the authors [31], this approach often gives more classes than the number of sources. This inevitably leads to artificial sources. Further thresholding is therefore suggested and its operation requires the a priori knowledge of the number of active sources in the system. As such information is likely to be unavailable in many applications, better classification techniques are required. To this end, the contribution in this work is to identify advanced clustering 
techniques which mitigate the problem of phantom sources and ideally are able to operate in the environment when the number of active sources is unknown.

\section{AdVANCED Clustering TeChNiQues FOR UNDERDETERMINED BSS}

The problem of classification refers to the issue of detecting the particular hidden structure in some data set $Z$. In our application of underdetermined BSS, $Z$ is formulated as the collection of eigenvectors $Z=\left\{\widetilde{\mathbf{v}}\left(t_{i}, f_{i}\right)\right\}$ obtained by eigen decomposition of the TF matrices $D_{\mathbf{x x}}\left(t_{i}, f_{i}\right)$ at different $\left(t_{i}, f_{i}\right)$. For a MIMO system of $k$ active sources, we expect these eigenvectors to be partitioned into $k$ subsets and that each of these subsets is associated with the TF representation of one active source. Various clustering methods can be applied for this scenario if $k$ is known a priori, such as the widely used k-means algorithm (see, e.g. [42], [43]), which allows all the eigenvectors to be iteratively classified into $k$ subsets.

When the value of $k$ is not known, however, relatively few approaches have been reported in the open literature. One of the review articles is given in [33]. Generally speaking, there are two types of methodology for tackling this problem. For the first one, the clustering procedure is repeated for many different numbers of clusters. By noticing that although the error measure ${ }^{1}$ decreases monotonically as the number of clusters increases, if the reduction of error measurement becomes much slower at a certain value, it suggests that a natural number of clusters has been reached. In comparison, the second approach is a splitting procedure. A cluster is split in two when a certain condition is satisfied. In correspondence with these two approaches, we introduce two active source selection algorithms for underdetermined BSS, both of which are based on advanced clustering techniques, namely the method using Gap statistics [34] and SSCL [35]. For notation simplicity, we denote these two advanced clustering-based underdetermined BSS algorithms as Algorithm I and Algorithm II, which will be elaborated upon in the following two subsections, i.e., Section IV-A and Section IV-B, respectively.

\section{A. Algorithm I}

In Algorithm I, a set of TF matrices $D_{\mathrm{xx}}\left(t_{i}, f_{i}\right)$ is computed at different TF points. For computational simplicity, we only consider the admissible TF set $\Omega=\left\{\left(t_{i}, f_{i}\right) \mid\left\|D_{\mathbf{x x}}\left(t_{i}, f_{i}\right)\right\|_{F}>\right.$ $\xi$ \}, where $\xi$ is an easily defined threshold, as will be shown in the simulation section, for ensuring sufficient signal energy and $\|\cdot\|_{F}$ denotes the Frobenius norm. The matrices $D_{\mathbf{x x}}\left(t_{i}, f_{i}\right),\left(t_{i}, f_{i}\right) \in \Omega$ are subsequently decomposed into a set of rank-1 matrices as in (7), resulting in the data set of eigenvectors $Z=\left\{\widetilde{\mathbf{v}}\left(t_{i}, f_{i}\right),\left(t_{i}, f_{i}\right) \in \Omega\right\}$. In order to find out the number of active sources in the mixture, we introduce the technique of Gap statistics which enables the automatic identification of $k$.

For two vectors $\widetilde{\mathbf{v}}\left(t_{1}, f_{1}\right)$ and $\widetilde{\mathbf{v}}\left(t_{2}, f_{2}\right)$ in $Z$, their similarity is measured by $\left\|\widetilde{\mathbf{v}}\left(t_{1}, f_{1}\right)-\widetilde{\mathbf{v}}\left(t_{2}, f_{2}\right)\right\|_{2}^{2}$. Assume the data set

\footnotetext{
${ }^{1}$ The "error measure" is used to characterize the dispersion within each cluster, such as the averaged pairwise distance defined in (10).
}

is partitioned into $k$ clusters $C_{1}, C_{2}, \ldots C_{k}$, where $C_{i}$ is the set containing the member indexes of the $i$ th cluster. The average pairwise distance within the cluster $C_{i}$ is given by

$$
\zeta_{i}=\left(1 / 2 n_{i}\right) \sum_{r, r^{\prime} \in C_{i}}\left\|\widetilde{\mathbf{v}}\left(t_{r}, f_{r}\right)-\widetilde{\mathbf{v}}\left(t_{r^{\prime}}, f_{r^{\prime}}\right)\right\|_{2}^{2}
$$

where $r$ and $r^{\prime}$ are different indexes contained in $C_{i}$, and $n_{i}$ is the number of vectors within this cluster. Taking all the $k$ clusters into account, the error measure in Gap statistics is defined as

$$
E_{k}=\sum_{i=1}^{k} \zeta_{i}
$$

The logarithm of the error measure $\log \left(E_{k}\right)$ is compared with that computed from a reference data set $Z^{\prime}$ which is drawn from an appropriate distribution. The natural number of clusters is then estimated as the value at which $\log \left(E_{k}\right)$ falls the farthest below the reference curve, as the word Gap in the name suggests. To generate the reference distribution which is crucial for the Gap test, we employ the scheme as in [34] to form a uniformly distributed reference feature according to the direction of the principal component of the data $Z$. The justification of this approach is given by [34, Theorem 1 and 2], where more detailed implementations can be found. For ease of implementation, we summarize the whole algorithm (Algorithm I) in Table I, where $E_{q l}^{\prime}$ in step 4 are the error measurements of the reference data, and for each $l, E_{q l}^{\prime}$ is calculated via (10).

Upon the introduction of Gap statistics in underdetermined BSS, not only the drawback of phantom sources in [31] is overcome but also the TF separation algorithm is now operating in a completely blind fashion, without the knowledge of the number of sources. The disadvantage of the proposed approach lies in the required additional computational complexity.

\section{B. Algorithm II}

In Algorithm II, the TF orthogonality assumption is also required, but the set of eigenvectors $Z$ is partitioned by SSCL. A set of prototypes $\mathbf{p}_{l}$, which are defined to characterize the clusters (sources in our problem) are competing to win as every member of $Z$ is included in the examination. Ideally, each cluster is associated with a prototype at its center. Hence, the problem of estimating the right number of sources is effectively the process of determining the number of prototypes. However, it is difficult to know a priori the number of sources (or prototypes) in our data set. If the number of prototypes is less than that of sources, at least one prototype might represent more than one source. As a result, none of the sources could be correctly identified. To avoid this problem, we employ the SSCL algorithm [35] in which an asymptotic property vector (APV) $\mathbf{f}_{l}$ is introduced to guide the learning of prototypes $\mathbf{p}_{l}$, so that each prototype will locate only one natural cluster even when the prototypes outnumber the clusters.

Suppose at some moment during the learning of $\mathrm{p}_{l}$, the member selected from $Z$ is $\widetilde{\mathbf{v}}\left(t_{i}, f_{i}\right)$. According to [35], the 
TABLE I

ALGORITHM I: GAP STATISTICS

1) Compute quadratic TF representation $D_{x x}(t, f)$ as in eqn. (5) and keep the admissible TF set $\Omega$.

2) Compute the eigen decomposition of the matrices $D_{x x}\left(t_{i}, f_{i}\right),\left(t_{i}, f_{i}\right) \in \Omega$ and collect the eigenvectors into the data set $Z$.

3) For varying number of clusters $q=1,2, \ldots, Q$, compute the error measurement $E_{q}$ using (10).

4) Generate a number $L$ of reference data sets using the method mentioned above (see [34]). Cluster each one with the $k$-means algorithm and compute the dispersion measures $E_{q l,}^{\prime}, l=1,2, \ldots, L$ and $q=1,2, \ldots, Q$. The Gap statistics are then estimated by $G_{q}=\frac{1}{L} \sum_{l=1}^{L} \log \left(E_{q l}^{\prime}\right)-\log \left(E_{q}\right)$.

5) To account for the sample error in approximating an ensemble average with $L$ reference distributions, the standard deviation is computed as $S_{q}=\left(\frac{1}{L} \sum_{l=1}^{L}\left(\log \left(E_{q l}^{\prime}\right)-\bar{l}\right)^{2}\right)^{1 / 2}$, where $\bar{l}=\frac{1}{L} \sum_{l=1}^{L} \log \left(E_{q l}^{\prime}\right)$. By defining $S_{q}^{\prime}=$ $S_{q} \cdot\left(1+\frac{1}{L}\right)^{1 / 2}$, the number of clusters $k$ is estimated as the smallest $q$ such that $G_{q} \geq G_{q+1}-S_{q+1}^{\prime}$.

6) With the number of clusters identified, use the $k$-means algorithm to partition $Z$ into $k$ sub-sets. The TF set of $\Omega$ is also correspondingly divided into $k$ sub-sets, $\Omega_{1}, \ldots, \Omega_{k}$.

7) Use the knowledge of $\Omega_{1}, \ldots, \Omega_{k}$ to obtain the estimates $\widehat{D}_{s_{i} s_{i}}(t, f), i=1, \ldots, k$. Use an adequate source synthesis procedure to estimate the source signal $s_{i}(t), i=1, \ldots, k$.

update rules of the APV $\mathbf{f}_{l}$ and the prototype $\mathbf{p}_{l}$ can be easily given as

$$
\begin{aligned}
\mathbf{f}_{l}^{\text {new }} & =\mathbf{f}_{l}+\frac{1}{n_{\mathbf{f}_{l}}} \alpha_{l}\left(\widetilde{\mathbf{v}}\left(t_{i}, f_{i}\right)-\mathbf{f}_{l}\right) \Psi\left(\mathbf{p}_{l}, \mathbf{f}_{l}, \widetilde{\mathbf{v}}\left(t_{i}, f_{i}\right)\right) \\
\mathbf{p}_{l}^{\text {new }} & =\mathbf{p}_{l}+\alpha_{l}\left(\widetilde{\mathbf{v}}\left(t_{i}, f_{i}\right)-\mathbf{p}_{l}\right) .
\end{aligned}
$$

Here $\alpha_{l}$ is written as

$$
\alpha_{l}=\left(1+\frac{\left\|\mathbf{p}_{l}-\widetilde{\mathbf{v}}\left(t_{i}, f_{i}\right)\right\|_{2}}{\left\|\mathbf{p}_{l}-\mathbf{f}_{l}\right\|_{2}}\right)^{-2} .
$$

The function $\Psi\left(\mathbf{p}_{l}, \mathbf{f}_{l}, \widetilde{\mathbf{v}}\left(t_{i}, f_{i}\right)\right)$ is used to characterize whether or not the input vector $\widetilde{\mathbf{v}}\left(t_{i}, f_{i}\right)$ is inside the neighborhood of $\mathrm{p}_{l}$, i.e.,

$$
\Psi\left(\mathbf{p}_{l}, \mathbf{f}_{l}, \widetilde{\mathbf{v}}\left(t_{i}, f_{i}\right)\right)= \begin{cases}1 & \text { if }\left\|\mathbf{p}_{i}-\mathbf{f}_{i}\right\|_{2} \geq\left\|\mathbf{p}_{i}-\widetilde{\mathbf{v}}\left(t_{i}, f_{i}\right)\right\|_{2} \\ 0 & \text { otherwise. }\end{cases}
$$

The quantity $n_{\mathbf{f}_{l}}$, as in (11), is the winning counter of $\mathbf{f}_{l}$ and it can be updated by

$$
n_{\mathbf{f}_{l}}^{\text {new }}=n_{\mathbf{f}_{l}}+\alpha_{l} \Psi\left(\mathbf{p}_{l}, \mathbf{f}_{l}, \widetilde{\mathbf{v}}\left(t_{i}, f_{i}\right)\right) .
$$

The learning rules guide $\mathbf{f}_{l}$ to shift toward the $\widetilde{\mathbf{v}}\left(t_{i}, f_{i}\right)$, if and only if $\widetilde{\mathbf{v}}\left(t_{i}, f_{i}\right)$ is in the neighborhood of $\mathbf{p}_{l}$. According to (13), the member $\widetilde{\mathbf{v}}\left(t_{i}, f_{i}\right)$ will have little influence on the learning of $\mathbf{p}_{l}$ if it is far from the neighborhood of $\mathbf{p}_{l}$, since $\alpha_{l}$ will thus approach to zero. Upon convergence of $\mathbf{p}_{l}, \mathbf{f}_{l}$ asymtotically approaches $\mathbf{p}_{l}$, with no more members within the data set eligible for the learning of $\mathrm{p}_{l}$ [35].

Without the help of $\mathbf{f}_{l}, \mathbf{p}_{l}$ is actually trying to take all the clusters it wins, and will locate, in the manner of an oscillation, at the center of these clusters [35] (thereby failing to locate at any one of these clusters). This implies that although $\mathrm{p}_{l}$ finally locates at only one cluster with the help of $\mathbf{f}_{l}$, there exists an extra cluster trying to pull $\mathbf{p}_{l}$ from its located cluster to the center. To quantify how much $\mathbf{p}_{l}$ is attracted by this extra cluster, a center property vector (CPV) $\mathrm{g}_{l}$ is defined [35], which simply takes the exact arithmetic mean of the input data points for which a prototype $\mathbf{p}_{l}$ has so far been the winner and is then updated with the $k$-means algorithm. The criterion, whether $\left\|\mathrm{p}_{l}-\mathrm{g}_{l}\right\|_{2}>\epsilon$, is then used to determine when one of the prototypes should be split into two to generate the extra cluster, where $\epsilon$ is a threshold. For non-Gaussian distributed clusters, if $\widetilde{\mathbf{v}}\left(t_{i}, f_{i}\right)$ is of dimension $m$, a simple way to determine $\epsilon$ is $(1 / 50)\left(\max \left(S l_{1}, S l_{2} \ldots S l_{m}\right)\right)$ [35], where $S l_{i}$ is the scale of the $i$ th coordinate (i.e. $\left.\mathrm{g}_{i}\right)$ in the $m$-dimensional feature space. When one prototype splits into two, one stays in its current location and the other is initialized at a distant location to avoid unnecessary competition between these two in the new learning. This is ensured by introducing a distant property vector (DPV) $\mathbf{r}_{l}$ [35]. Its update equation is given by

$$
\mathbf{r}_{l}^{\text {new }}=\mathbf{r}_{l}+\frac{1}{n_{\mathbf{r}_{l}}} \beta_{l}\left(\widetilde{\mathbf{v}}\left(t_{i}, f_{i}\right)-\mathbf{r}_{l}\right) \Psi\left(\mathbf{p}_{l}, \widetilde{\mathbf{v}}\left(t_{i}, f_{i}\right), \mathbf{r}_{l}\right)
$$

where $n_{\mathbf{r}_{l}}$ is the learning counter for $\mathbf{r}_{l}$ and $\beta_{l}$ is given by

$$
\beta_{l}=\left(1+\frac{\left\|\mathbf{p}_{l}-\mathbf{r}_{l}\right\|_{2}}{\left\|\mathbf{p}_{l}-\widetilde{\mathbf{v}}\left(t_{i}, f_{i}\right)\right\|_{2}}\right)^{-2} .
$$

This learning procedure algorithm continues until no further source is suggested by the splitting criterion. In summary, the essential steps of the Algorithm II for active source selection in underdetermined BSS are listed in Table II.

Compared with Algorithm I, the advantage of Algorithm II is in its capability of automatically determining the number of clusters in the data set and moreover its computational simplicity when dealing with a large data set. However, if the size of data set $Z$ is small, Algorithm I is more applicable, as it avoids the procedure of adaptive learning as in Algorithm II. More specifically, if the size of $Z$ is denoted as $N_{z}$, the required computation (multiplications and additions) for Algorithm I is approximately $\left[2\left(m N_{z}+k\right)(1+L)+(3 L+2)\right] Q$ (without the operations for generating the data set $Z$ ), and for Algorithm II, approximately $\left(15 m+N_{z}+11\right) k$. The performance evaluation of these two new algorithms will be given in Section VI. 
TABLE II

ALGORITHM II: SSCL

1) Conduct the first two steps as in Algorithm I to get the data set $Z$.

2) Set the number of clusters $k=1$; initialize $p_{l}=r_{l}$ at some location in the feature space; initialize $f_{l}=g_{l}$ at some location far away from $p_{l}$; all counters $=0$.

3) Repeat the following process until $\left\|p_{l}-f_{l}\right\|_{2}<\epsilon$ or a predetermined number of iterations has been reached.

4) Randomly take a sample $\widetilde{v}\left(t_{i}, f_{i}\right)$ from $Z$ and find the winning prototype $p_{l}$ based on $p_{l}=\arg \min _{j}\left\|p_{j}-\widetilde{v}\left(t_{i}, f_{i}\right)\right\|_{2} ;$ allocate $\widetilde{v}\left(t_{i}, f_{i}\right)$ to cluster-l;

5) Update $\mathrm{APV} f_{l}$ using eqn (11), and update $\mathrm{CPV} g_{l}$ using the $k$-means algorithm;

6) Update DPV $r_{l}$ using eqn (16), and update prototype $p_{l}$ using eqn (12);

7) If $\max _{l}\left\|p_{l}-g_{l}\right\|_{2}<\epsilon$, find $k$ clusters and the TF set $\Omega$ is consequently partitioned into $k$ subsets, namely $\Omega_{1} \ldots \Omega_{k}$. Otherwise, increase $k$; set $p_{k}$ as $r_{l}$; go to step 3 .

8) Use the knowledge of $\Omega_{1} \ldots \Omega_{k}$, to obtain the estimates $\widehat{D}_{s_{i} s_{i}}(t, f), i=1 \ldots k$. Use an adequate source synthesis procedure to estimate the source signal $s_{i}(t), i=1 \ldots k$.

\section{ON THE VIOLATION OF THE TF ORTHOGONALITY ASSUMPTION}

The assumption of TF orthogonality is employed in Section IV to facilitate the solution for underdetermined BSS. This orthogonality assumption is in fact commonly required in most of the underdetermined bss methods that exploit sparsity in the TF domain [26]-[31]. In a more general case, however, it is likely that there exists an overlap between the TF distributions of different sources. As will be demonstrated in Section VI for the GMSK signals, even if the carrier frequency is well separated, overlap in the TF domain prevents successful source separation when multiple sources are simultaneously present in the system. In this sense, we are motivated to search for more practical algorithms.

Note that the importance of the TF assumption lies in the fact that it enables the quadratic representation $D_{\mathbf{x x}}\left(t_{i}, f_{i}\right)$ to be written as a rank one matrix. That is, if only source- $j$ is present at $\left(t_{i}, f_{i}\right)$ in the system, $D_{\mathbf{x x}}\left(t_{i}, f_{i}\right)$ can be written as a rank one matrix, i.e. (7). The principal eigenvector coincides with the steering vector subject to a random phase difference. But in the violation of the assumption, the TF matrix $D_{\mathbf{x x}}\left(t_{i}, f_{i}\right)$ is no longer a rank one matrix as in (7) and the principal eigenvector of the matrix $D_{\mathbf{x x}}\left(t_{i}, f_{i}\right)$ is no longer colinear with the steering vector $\mathbf{a}_{j}$. Partitioning the set of principle eigenvectors into $k$ clusters therefore becomes meaningless and therefore leads to failure of source separation.

In this paper, we relax the TF orthogonal assumption as follows.

Assumption 1: At any time instant, the number of active sources $d$ does not exceed the number of antennas, i.e., $0 \leq d \leq m .^{2}$

Following the relaxation of the orthogonality assumption, $D_{\mathbf{x x}}(t, f)$ is no longer a rank-1 matrix. As there is no clear relationship between the eigenvectors of $D_{\mathbf{x x}}(t, f)$ and the

${ }^{2}$ We do not consider the case $d>m$ in this work as this has already been demonstrated in, for example, [17], [18], [45], [46], to cause algorithm breakdown. steering vectors, using singular value decomposition (SVD) on $D_{\mathbf{x x}}(t, f)$, the only information obtained is the number of active sources at that specific point. But which $d$ out of $k$ sources are active is difficult to identify. However, in many applications, it is reasonable to assume that a signal is likely to exist in the system for a certain minimum length of time once detected. In other words, we assume certain temporal continuity of the input sources. This can be justified for example in communications applications, where the sources are nonstationary in the sense of their sudden arrival or departure. Thus in a short time interval, a fixed number of sources is present in the system and this number does not exceed the number of the antennas $m$. Based upon this property, the basic idea of our approach is to cluster the neighboring TF points that correspond to the same active sources. As a result, the whole TF plane is partitioned into a certain unknown number of blocks. In each block, at most $m$ sources exist and the adjacent blocks contain different numbers of sources. The important point here is that, within the same block, the active sources remain unchanged. Signal processing can therefore be carried out in a block-by-block manner. Moreover, since there are at most $m$ sources present in each block, the problem of underdetermined BSS is transformed into exactly or over determined blind source separation, for which there are known solutions. It is of interest to note that Assumption 1 is implicitly guaranteed with the help of the block-based operation and the exploitation of the introduced advanced cluster techniques, which make the block-based operation possible. This assumption also agrees with some application scenarios, as verified for communication signals examined in our simulations. In contrast to the widely used sparsity assumption, our assumption is less restrictive in the sense that we do not assume any probability distribution models of the sources.

In practice, one of the major difficulties is in the way that the TF plane is partitioned. Consider a three sources and two antennae system as an example. With an SVD applied to every TF point $\left(t_{i}, f_{i}\right)$, we obtain 
TABLE III

ALGORITHM III: BLOCK-BASED TF PARTITIONING APPROACH

1) Initialization: $\Gamma_{1}=\emptyset, \cdots, \Gamma_{m}=\emptyset$. That is, $\Gamma_{l}$ is an empty set which is going to contain $\left(t_{i}, f_{i}\right)$ associated with the presence of a number $l$ of active sources.

2) Compute quadratic TF representation $D_{x x}(t, f)$ as in eqn. (5) and keep the admissible TF set $\Omega$.

3) For $\forall\left(t_{i}, f_{i}\right)$ in $\Omega$, compute its eigen decomposition $D_{x x}\left(t_{i}, f_{i}\right)=V \Lambda\left(t_{i}, f_{i}\right) V^{-1}$, where $\Lambda\left(t_{i}, f_{i}\right)=$ $\operatorname{diag}\left(\lambda_{1} \ldots \lambda_{m}\right)$. If $\lambda_{1}>\cdots>\lambda_{l} \gg \lambda_{l+1}>\cdots>\lambda_{m},\left(t_{i}, f_{i}\right)$ is assigned to the set $\Gamma_{l}$, i.e., $\Gamma_{l}=\Gamma_{l} \cup\left(t_{i}, f_{i}\right)$.

4) For the non-empty set $\Gamma_{2}, \cdots, \Gamma_{d}, d \leq m$, apply the SSCL algorithm or the method based on Gap statistics to group the neighbouring T-F points into blocks, e.g., $\Gamma_{l}=\Gamma_{l}^{(1)} \cup \Gamma_{l}^{(2)} \cdots \cup \Gamma_{l}^{(\hbar)}$. That is, the set $\Gamma_{l}$ is divided into subsets $\Gamma_{l}^{(1)}, \cdots, \Gamma_{l}^{(\hbar)}$, where $\hbar$ is unknown.

5) For overlapped blocks $\Gamma_{l}^{(1)}, \ldots, \Gamma_{l}^{(h)}, l=2 \ldots d$, apply the JADE algorithm (see [1]) to obtain $\hat{s}$ and a set $A$ of steering vectors at the specified block.

6) For the block during which only one source is present, $\Gamma_{1}$, apply the FOCUSS algorithm (see [23]) or the algorithm in section IV to obtain $\hat{s}$ and a set $A^{\prime}$ of steering vectors at the specified block.

7) For the steering vectors $A$ and $A^{\prime}$, use the $k$-means algorithm to classify them into $k$ clusters, the center vector of each cluster is the estimated steering vector $\hat{a}_{i}$, where $i=1, \cdots, k$.

8) According to the same steering vector, line up the signal $\hat{s}$ from different blocks.

set $\Gamma_{1}=\left\{\left(t_{i}, f_{i}\right) \mid\right.$ single active source present $\}$ and $\Gamma_{2}=\left\{\left(t_{i}, f_{i}\right) \mid\right.$ two active sources present $\}$. It is, however, difficult to partition $\Gamma_{1}$ and $\Gamma_{2}$ into appropriate blocks, each of which is contributed to by the same set of active sources. For example, a subset of $\Gamma_{2}$ may be contributed to by source-1 and source-2, while another subset of $\Gamma_{2}$ may be contributed to by source- 2 and source- 3 . As it is nearly impossible to know the number of such subsets a priori, when grouping the neighboring TF points using classification methods, an advanced clustering technique which does not require the knowledge of the number of clusters is needed. The methods based on Gap statistics and self-splitting competitive learning are, therefore, suitable candidates for the operation.

With these advanced clustering techniques, the TF plane is expected to be divided into blocks, during which the same sources are present. A conventional BSS algorithm can subsequently be applied for each block. There are various choices for the conventional BSS algorithm. For example, here the JADE algorithm [1] is applied. The active sources in every time interval are estimated together with the associated steering vectors. However, due to the inherent permutational ambiguity of BSS algorithms, the problem of lining up the blocks that correspond to the same source is raised. To tackle this problem, notice from (1) that each source $s_{i}(t)$ is associated with a steering vector $\mathbf{a}_{i}$, which is assumed unchanged for the whole observation period. Hence, by classifying the set of estimated steering vectors using, for example, the conventional $\mathrm{k}$-means algorithms, the permutational problem is solved. To summarize, our proposed robust algorithm (denoted as Algorithm III) considering the TF orthogonality assumption violation in underdetermined BSS is conducted as in the steps listed in Table III.
With our block-based algorithm, not only the sources with overlap between their TF distributions can be separated, but the problem of phantom sources is mitigated. An additional merit is that the algorithm performs totally blindly without the knowledge of the number of active sources. However, a disadvantage of our approach is the computational complexity introduced by the TF representation and block-based operation. Additionally, although the orthogonality assumption has been relaxed, our proposed algorithm should be applied with the restriction of Assumption 1 for the achievement of good separation performance. Another issue should be clarified that due to the advanced clustering techniques being introduced, the boundaries of the blocks are automatically determined and vary with different blocks. It is unlikely to be able to manually select a fixed boundary suitable for all TF blocks, and in a real problem a fixed boundary would be prohibitive. It is therefore an advantage to introduce the advanced clustering techniques for the automatic determination of the boundaries of these blocks, which thereby enhances the feasibility of the proposed block-based algorithm.

It should be noted that there exist other interesting approaches for tackling the underdetermined BSS problem, such as the minimum $l_{1}$-norm approach [45], geometric approach [46], and algebraic approach [18]. A common feature with these approaches is that the mixing matrix $A$ and source signals $\mathbf{s}$ are estimated separately or in an iterative procedure, where $A$ is estimated under the help of a distribution model (e.g. the assumption of Laplacian prior) of the original sources captured from the observed mixtures, and $\mathbf{s}$ is estimated through the maximum a posteriori probability (MAP) approach [45]. One of the attractive properties with these approaches is their relative ease of implementation [46]. However, the geometric approach requires a relatively large number of iterations for convergence [46], the algebraic approach requires the assumption that the dimension of $A$ is known [18], and the separation performance for dif- 
TABLE IV

Number of Clusters Found Versus the Value of Threshold for the Linear Chirp Signals

\begin{tabular}{c|c|c}
\hline \hline Threshold $\varepsilon$ & No. of clusters found & Successful separation(Yes/No) \\
\hline 0.4 & 1 & $\mathrm{~N}$ \\
0.2 & 3 & $\mathrm{~N}$ \\
0.1 & 9 & $\mathrm{~N}$ \\
0.05 & 23 & $\mathrm{Y}$ \\
\hline \hline
\end{tabular}

TABLE $\mathrm{V}$

Number of Clusters Found Versus the VALUe of Threshold For the GMSK Signals

\begin{tabular}{c|c|c}
\hline \hline Threshold $\varepsilon$ & No. of clusters found & Successful separation(Yes/No) \\
\hline 0.4 & 2 & $\mathrm{~N}$ \\
0.3 & 3 & $\mathrm{~N}$ \\
0.2 & 4 & $\mathrm{~N}$ \\
0.1 & 7 & $\mathrm{Y}$ \\
\hline \hline
\end{tabular}

ferent mixing matrices is not always stable [45]. In contrast, our approach exploits the nonstationarity of sources without the requirement of prior probability distribution knowledge of sources. Additionally, due to its block operation, the problems of instability caused by the mixing matrices and requirement of long data samples for convergence are likely to be overcome with our approach. Due to the different framework being taken, we compare our algorithms with that of [31] since it is also a TF representation-based approach.

\section{NUMERICAL EXPERIMENTS}

\section{A. Performance Evaluation of Algorithm I and II}

Here, the performance evaluation of the proposed approaches will be compared with the method in [31] (see also the discussion in Section III). A MIMO system with three sources $(k=3)$ and two sensors $(m=2)$ is assumed. A mixing matrix is assumed of the form

$$
A=\left[\begin{array}{ccc}
0.4+0.1 j & 0.9+0.2 j & 0.6+0.5 j \\
0.6+0.6 j & 0.7+0.2 j & 0.4+0.3 j
\end{array}\right] .
$$

Additive white Gaussian noise at a level of $20 \mathrm{~dB}$ signal-to-noise ratio (SNR) is assumed. It should be noted that the purpose of adding a certain level of noise in the mixtures is to demonstrate the reasonable robustness obtained with our proposed algorithms. This property is a result of the exploitation of the TF representation, where the source energy is effectively localized with the noise power spreading over the whole TF plane [10]. However, the focus of this work is to demonstrate the feasibility of our proposed approaches, more extensive evaluations on different levels of noise using SNR, Eb/N0 (normalized SNR), or bit-error-rate (BER) are therefore discounted in this study. Instead, TF plots are used in common with many published works in the field, such as [31]. To reduce the cross term interference, the quadratic TF representation is selected as the Choi-William distribution [44], which belongs to the category of Cohen's Class of TF distribution [32]. We use the TF toolbox for MATLAB [40] to generate the TF representation of signals. In the initialization, we set $\mathbf{p}_{1}$ to a random location in
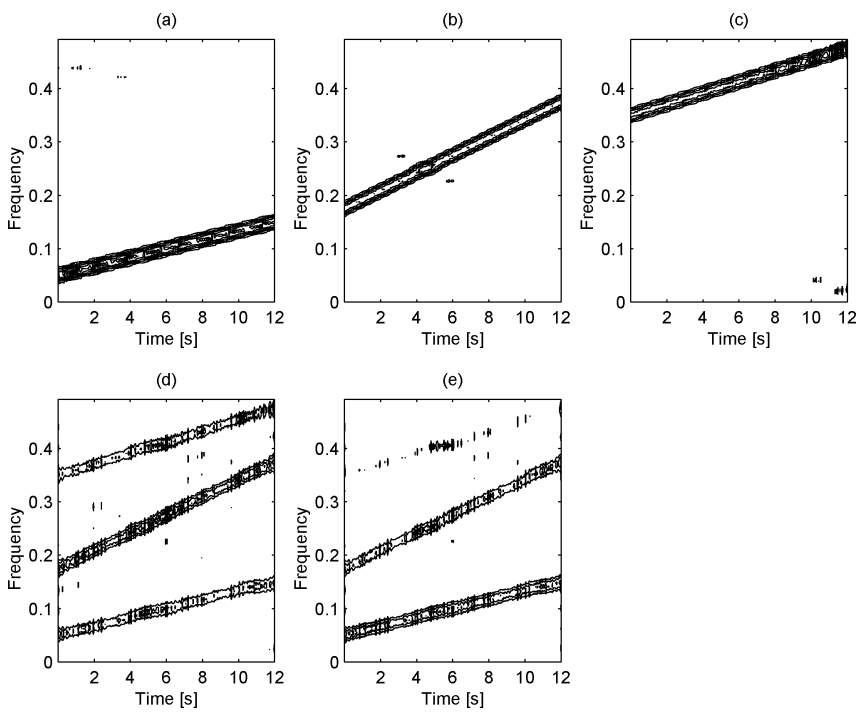

Fig. 1. Simulation with linear chirp signal. (a) Source-1. (b) Source-2. (c) Source-3. (d) Received mixture-1. (e) Received mixture-2.

the input space, $\left\|\mathbf{f}_{1}-\mathbf{p}_{1}\right\|_{2}>3$ to ensure $\mathbf{f}_{1}$ is far away from $\mathbf{p}_{1}, \mathbf{g}_{1}=\mathbf{f}_{1}, \mathbf{r}_{1}=\mathbf{p}_{1}$, and $n_{\mathbf{f}}=n_{\mathbf{r}}=n$. To obtain admissible TF set $\Omega$, we set the threshold $\xi$ to be 10 . Two types of signals are used in the experiments, i.e. linear chirp signals and GMSK modulated signals, respectively.

1) Linear Chirp Signals: For a linear chirp signal, its instantaneous frequency varies linearly with time. At time $t$, a monocomponent linear chirp signal $s_{j}(t)$ can be expressed as

$$
s_{j}(t)=\cos \left(2 \pi f t+\theta_{0}\right) .
$$

Here, we assume that at time $t_{0}$, the instantaneous frequency of $s_{j}(t)$ is $f_{0}, \theta_{0}$ is the initial phase and the instantaneous frequency is given by $f=f_{0}+\triangle f_{t}\left(t-t_{0}\right)$, where $\triangle f_{t}$ is a linear slope parameter. The TF representations of the source signals and the two received mixtures are shown in Fig. 1 where the frequencies are normalized, and this style of plot is used throughout this section. Using the method in [31], when the value of $\varepsilon$, in (9) is gradually reduced from 0.4 to 0.05 , the number of clusters 


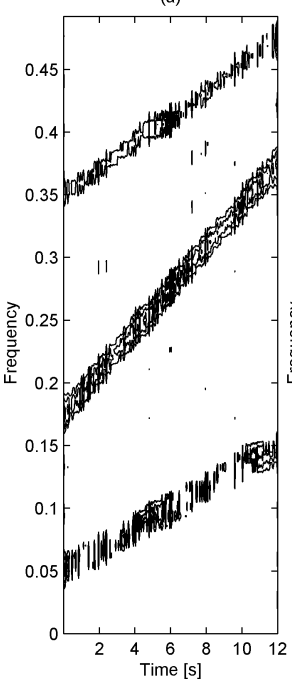

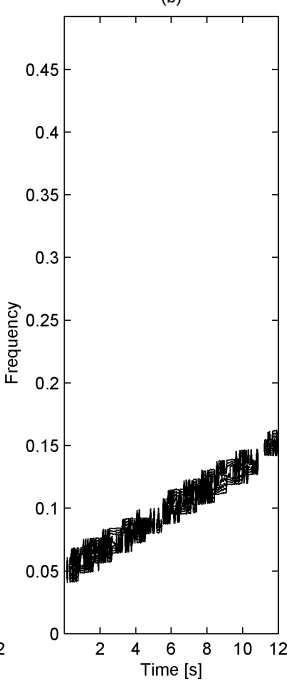

(c)
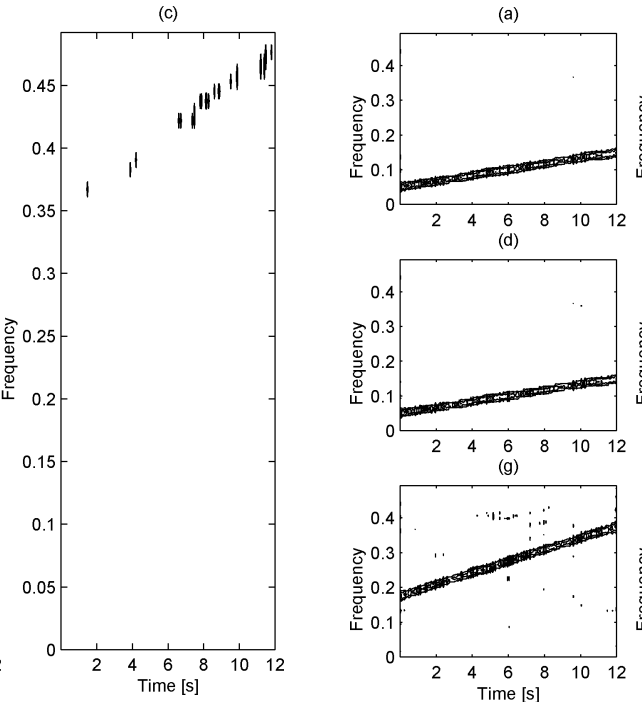

(b)

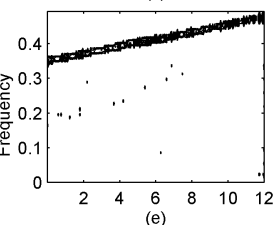

(e)
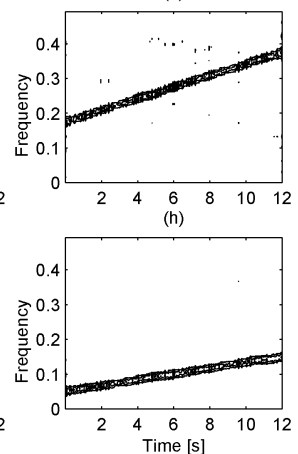
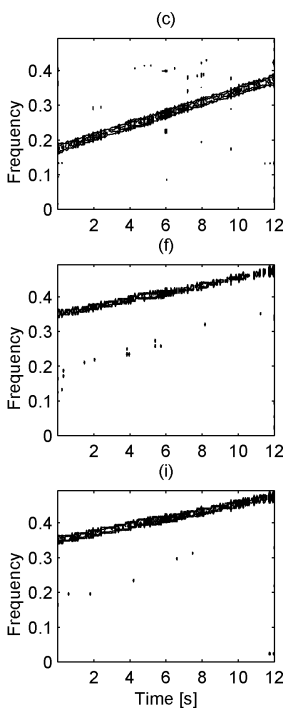

Fig. 2. Unsuccessful separation using simple classification method with linear chirp signals. (a) Unmixing output-1. (b) Unmixing output-2. (c) Unmixing output-3.

found is shown in Table IV. Note that, Table IV, and the subsequent Table $\mathrm{V}$ are all the simulation results by using method [31] to demonstrate that it fails either to give the right number of sources (resulting in phantom sources) or to give successful separation of the sources. However, since Algorithm I and II can give exactly the right number of sources and successful separation for all the thresholds $\varepsilon$ in the tables, similar tables are omitted without ambiguities. The results show that when $\varepsilon$ is selected as 0.2 , three clusters are suggested. This matches the natural number of the input sources. However, source separation is not successful, as depicted by the TF representation of the unmixing outputs in Fig. 2. In particular Fig. 2(b) shows successful extraction of source-1, but both Fig. 2(a) and (c) demonstrate failure to extract source-2 and source-3. For successful source separation, the threshold value should decrease (e.g., $\varepsilon=0.05$ in Table IV), which, however, leads to the unavoidable artificial sources (e.g., in total 23 sources were found). The simulation results of using advanced clustering techniques are shown in Fig. 3. Successful source separation is confirmed in the simulations. Comparing the separation results, we know that although both the $k$-means algorithm and the algorithm in [31] assume the true number of clusters is known, the former appears to perform much better than the latter one. Moreover, with the $k$-means algorithm, the threshold value $\varepsilon$ in (9) does not need selecting and the drawback of phantom sources is overcome. In practical situations, the number of clusters is often unknown a priori. This problem can be effectively removed by the proposed advanced clustering methods. For Algorithm I, the Gap statistics method is applied before using the $k$-means algorithm. The natural number of clusters is reached when the gap between the actual data and the reference data is largest. This can be confirmed in Fig. 4. Twenty copies of reference data, i.e., $L=20$, are generated in the simulation. The corresponding unmixing outputs are shown in Fig. 3(d)-(f). The separation results of Algorithm II are shown in Fig. 3(g)-(i). The algorithm capability of separating more sources than sensors is therefore confirmed even when the number of active source is unknown to the observer.
Fig. 3. Successful source separation with linear chirp signal. (a)-(c) Unmixing signals with the k-means algorithm. (d)-(f) Unmixing signals with Algorithm I without knowing the number of sources. (g)-(i) Unmixing signals with Algorithm II without knowing the number of sources.

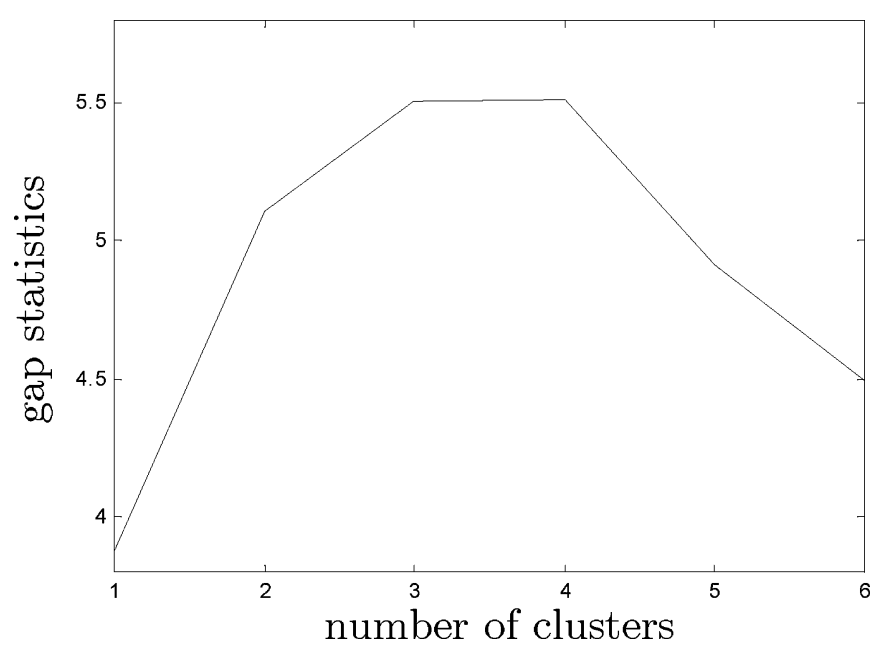

Fig. 4. Gap statistics in clustering with linear chirp signals. In this case, the largest gap happens when the number of clusters equals to 3 .

2) GMSK Signals: The monocomponent linear chirp signals employed in the simulations are the simplest nonstationary signals in the sense that their instantaneous frequency content consists of only a single frequency. Therefore, provided that the instantaneous frequencies of the input sources differ, the TF orthogonality assumption required in underdetermined BSS is readily satisfied even though all sources might be simultaneously present within the measurements.

For a GMSK modulated source, which is used in a GSM communication system, its spectrum is no longer a single frequency component but a portion of frequencies whose bandwidth depends on the signal data rate and normalized bandwidth of the Gaussian filter. As a result, although this kind of signal is praised for its compact spectrum (i.e., the side lobes of the GMSK are low and the main lobe is narrow compared with that using a rectangular pulse), when multiple sources are present within the measurements at the same time, the TF supports of the sources 

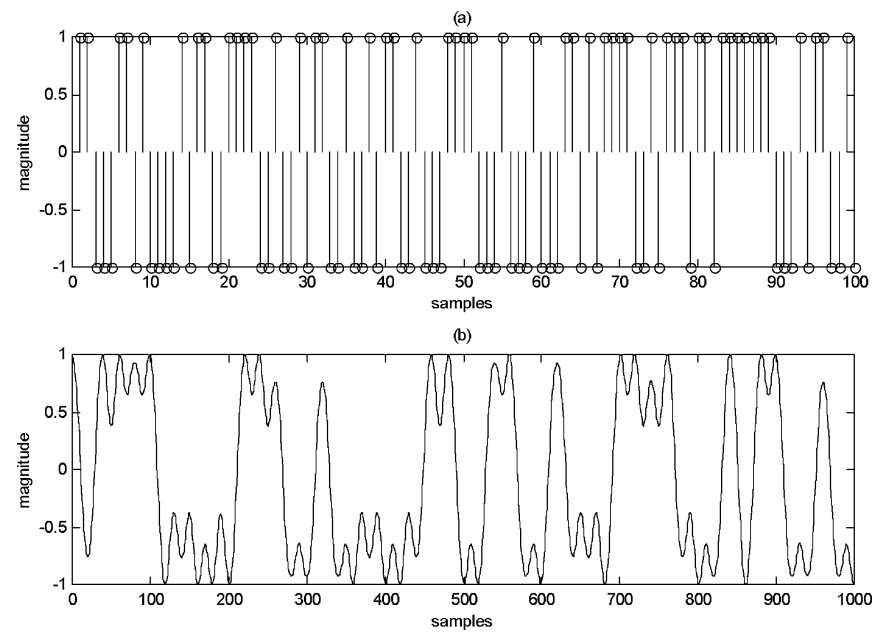

Fig. 5. GMSK modulated signals. (a) Sampled data. (b) In-phase data of GMSK modulated signal.

are likely to be overlapped if the gap between the carrier frequencies is small. This possible overlap in the TF domain violates the orthogonality assumption and may lead to failure in source separation, since the principle eigenvector of the matrix $D_{\mathbf{x x}}(t, f)$ is no longer the steering vector of a source (i.e., not a column vector of the mixing matrix $A$ ). Simulation results under this scenario will be demonstrated next.

Assume that the data rate of the sources is $1000 \mathrm{bits} / \mathrm{s}$ and the sampling rate is $10 \mathrm{ksamples} / \mathrm{s}$. Some of the data samples from source-3 are shown in Fig. 5(a). The corresponding in-phase data channel of the GMSK modulated signal is shown in Fig. 5(b). The normalized bandwidth of the Gaussian filter is selected as 0.3 . In the first simulation, we assume that the input sources are presented in the measurements at different time intervals. The carrier frequency is $3 \mathrm{kHz}$, which is the same for all three sources. Note that, compared with the realistic GSM system, where the bit rate at the physical layer is $270 \mathrm{~Kb} / \mathrm{s}$ and the carrier frequency is 900 or $1800 \mathrm{MHz}$, the relatively low data rates and radio frequencies are chosen in our experiments for the reduction of the run-time and computational cost of the simulations. The algorithm was developed without constraints on frequency range, which means the results for higher frequencies and data rates would essentially be the same as we have shown here. With the same mixing matrix as in (18), the source signals and the received mixtures are shown in Fig. 6. By choosing different values of the threshold $\varepsilon$, the number of clusters obtained is shown in Table V. The simulations results are similar to those of linear frequency modulated signals. The sensitivity in the selection of the threshold value is once again confirmed. In comparison, empirical selection of such a threshold is overcome by Algorithm I and Algorithm II, both of which are capable of extracting all three sources without the knowledge of the number of active sources. The results are depicted in Fig. 7. The Gap statistics, which are employed in Algorithm-1, are shown in Fig. 8. The number of active sources in the system is correctly identified. From these simulations, it follows that the proposed Algorithm I and Algorithm II outperform the existing approach with the TF orthogonality assumption. For the violation of this assumption, experimental results we obtained agree with the theoretical formulations in
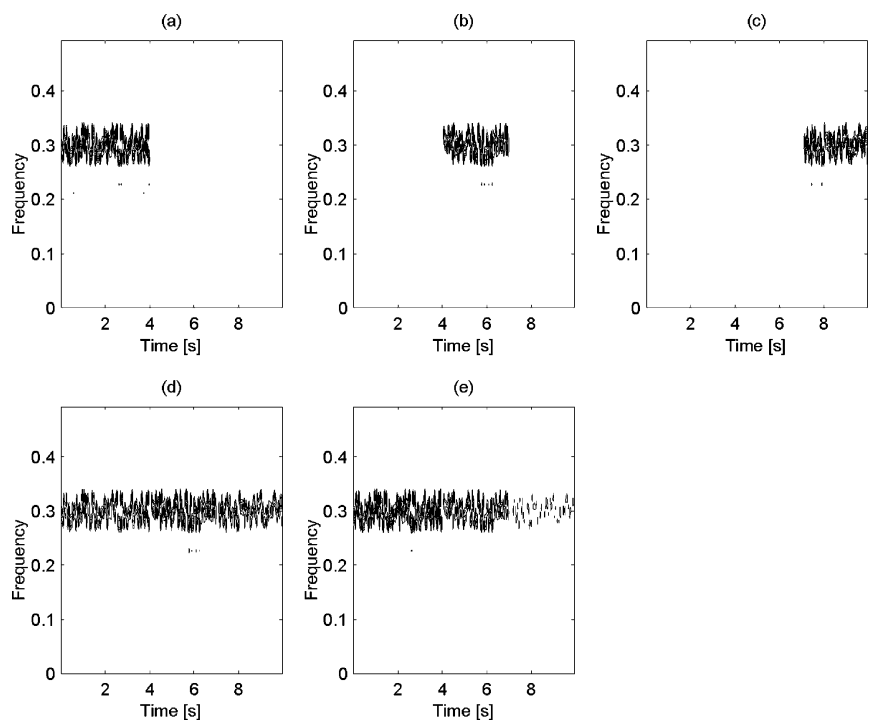

Fig. 6. Simulation with GMSK. (a) Source-1. (b) Source-2. (c) Source-3. (d) Received mixture-1. (e) Received mixture-2.
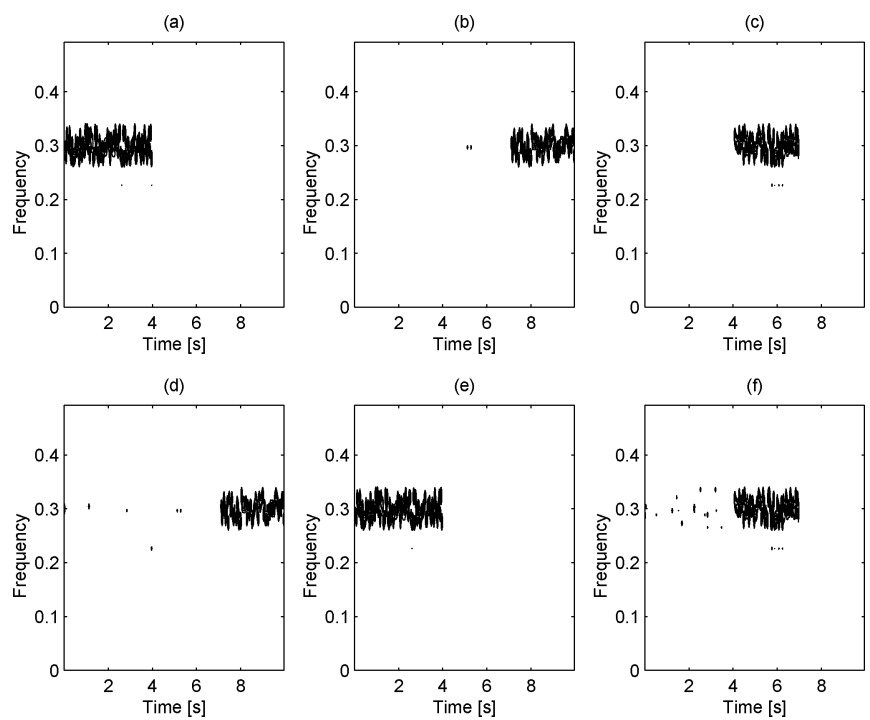

Fig. 7. Successful source separation with GMSK signal. (a)-(c) Unmixing signals with Algorithm I without knowing the number of sources. (d)-(f) Unmixing signals with Algorithm II without knowing the number of sources.

Section IV which suggest that all the algorithms examined in this section cannot successfully separate the sources.

\section{B. Implementation Example of Algorithm III}

The simulation is put into the context of blind beamforming. Assume a three GMSK source and two sensor system. The two measurement sensors are separated by half of the wavelength. The directions of arrival (DOAs) of the three sources are assumed to be $0, \pi / 9$, and $2 \pi / 3$, respectively. The mixing matrix is thus written as

$$
\begin{aligned}
A & =\left[\begin{array}{ccc}
1 & 1 & 1 \\
e^{-j \pi \sin (0)} & e^{-j \pi \sin \left(\frac{\pi}{9}\right)} & e^{-j \pi \sin \left(\frac{2 \pi}{3}\right)}
\end{array}\right] \\
& =\left[\begin{array}{ccc}
1 & 1 & 1 \\
1 & 0.4762-0.8793 i & -0.9127-0.4086 i
\end{array}\right] .
\end{aligned}
$$




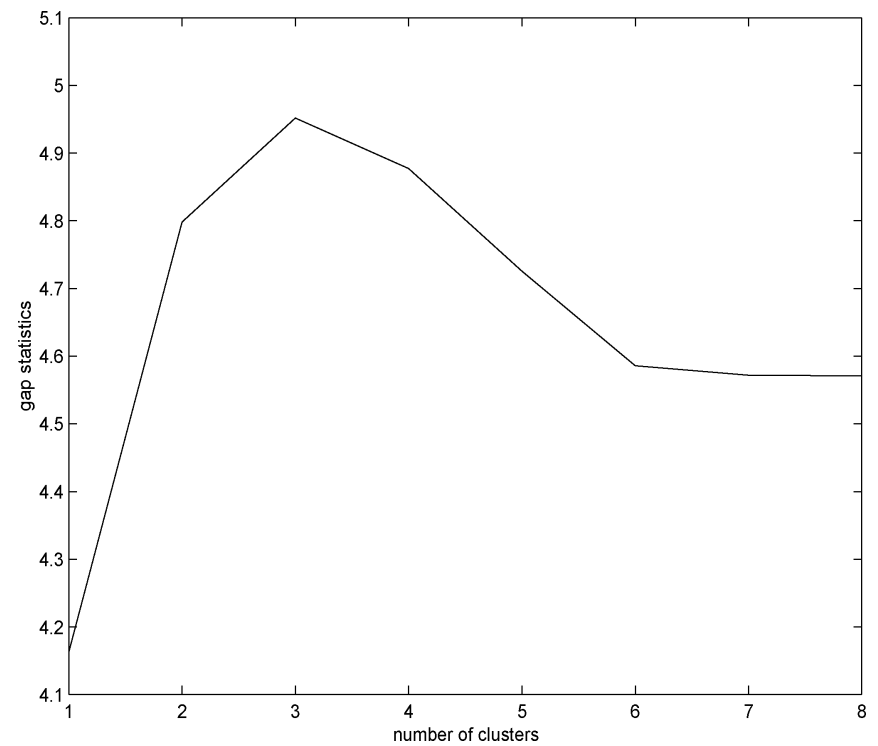

Fig. 8. Gap statistics in clustering with GMSK modulated signals.

TABLE VI

DESCRIPTION OF THREE NON-STATIONARY SOURCE SignaLS

\begin{tabular}{c|c}
\hline \hline Source No. & Present time in the system \\
\hline Source 1 & $0-4000 \mathrm{sec}, 5001-7000 \mathrm{sec}, 8001-10000 \mathrm{sec}$ \\
Source 2 & $2001-5500 \mathrm{sec}$ \\
Source 3 & $7101-10000 \mathrm{sec}$ \\
\hline \hline
\end{tabular}

Note that, the mixing matrix was selected to be in common with the standard formulation of DOA estimation, where the first sensor has been implicitly set as the origin of the linear array, and the amplitude response has been omitted for the sake of simplicity. However, there is no restriction with the form of mixing matrix which can have all complex entries since our algorithm has been developed for general mixing matrices (there is only one phase difference for complex numbers as discussed in Section III). The data rate is $1000 \mathrm{bits} / \mathrm{s}$ and the sampling rate is $10 \mathrm{ksamples} / \mathrm{s}$. The carrier frequencies of the three signals are set to 3000, 3500, and $4000 \mathrm{~Hz}$. The active times of different source signals are summarized as in Table VI and their TF representations are shown in Fig. 9(a)-(c). The two received mixtures are shown in Fig. 9(d) and (e). It can be observed that the overlap regions will be over time interval 2001-4000 s, 5001-5500 s, and 8000-10000 s. By testing the rank of the TF matrices at different TF points, the regions at which two sources are present are successfully identified and shown in Fig. 10(a). The corresponding nonoverlap regions are also successfully identified, as shown in Fig. 10(b). To estimate the input signals and the associated steering vectors, first we consider the overlap regions. By classifying the $\mathrm{TF}$ points that are associated with the presence of two active sources using the SSCL algorithm, the overlap region in Fig. 10(a) is partitioned into three TF blocks. They are described, respectively, in Fig. 11. In the simulation, for the block in Fig. 11(a), the estimated time interval is $8020-10000 \mathrm{~s}$. The estimated time interval for Fig. 11(b) is 2020-4020 s and that for Fig. 11(c) is 5021-5521. Recall from
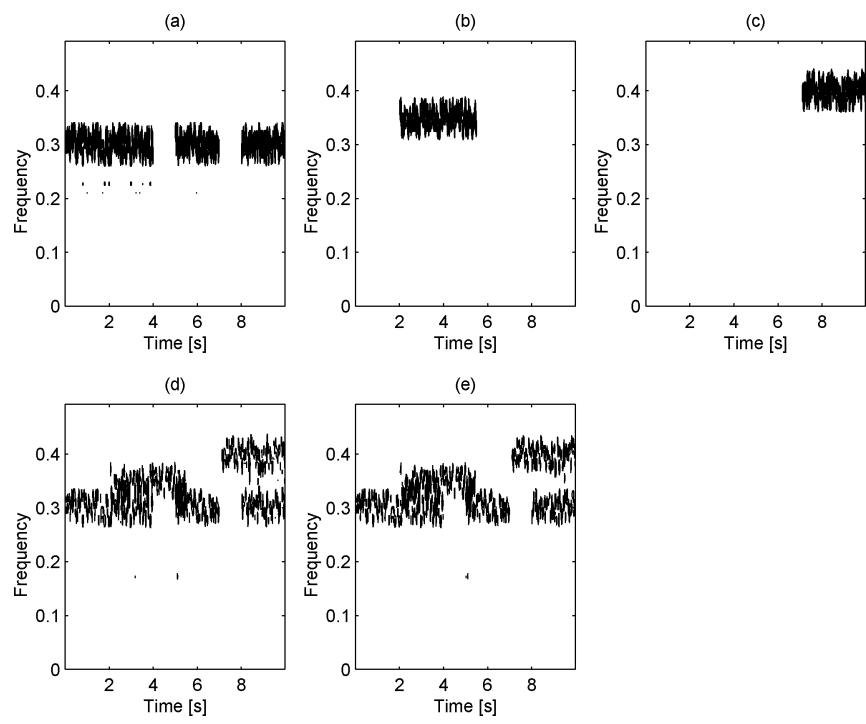

Fig. 9. In the violation of time-frequency orthogonality assumption. (a) Source-1. (b) Source-2. (c) Source-3. (d) Received mixture-1. (e) Received mixture-2.
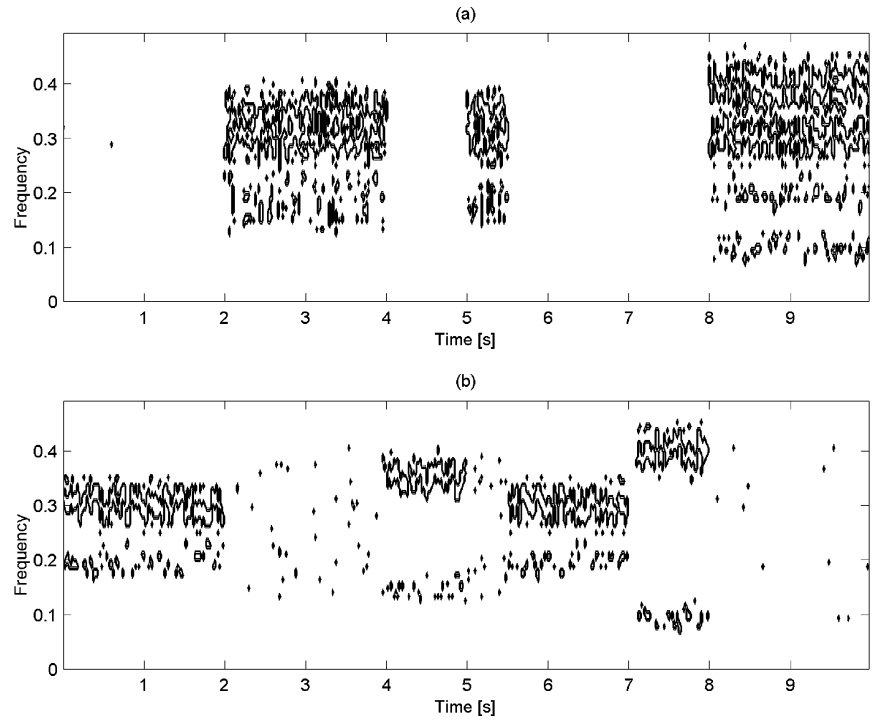

Fig. 10. Separation of overlap and nonoverlap regions.

the simulation setting that there are three individual time intervals, namely 2001-4000 s, 5001-5500 s, and 8000-10 $000 \mathrm{~s}$, in which two out of three sources (we do not know which two yet) are present. The experimental results are all in close agreement with the actual boundaries. In this sense, if the nonstationarity of the sources is in their sudden arrival and departure, it is possible to divide the whole observation time into appropriate time intervals with the help of the TF information. After successful identification of the overlap regions, the conventional JADE algorithm is applied to estimate the corresponding steering vectors and the input sources in each block. For TF block shown in Fig. 11(b), with the JADE algorithm, the two steering vectors are estimated as

$$
\left[\begin{array}{ll}
\hat{\mathbf{a}}_{1} & \hat{\mathbf{a}}_{2}
\end{array}\right]=\left[\begin{array}{cc}
1 & 1 \\
1.0096+0.0268 i & 0.4575-0.9018 i
\end{array}\right] .
$$



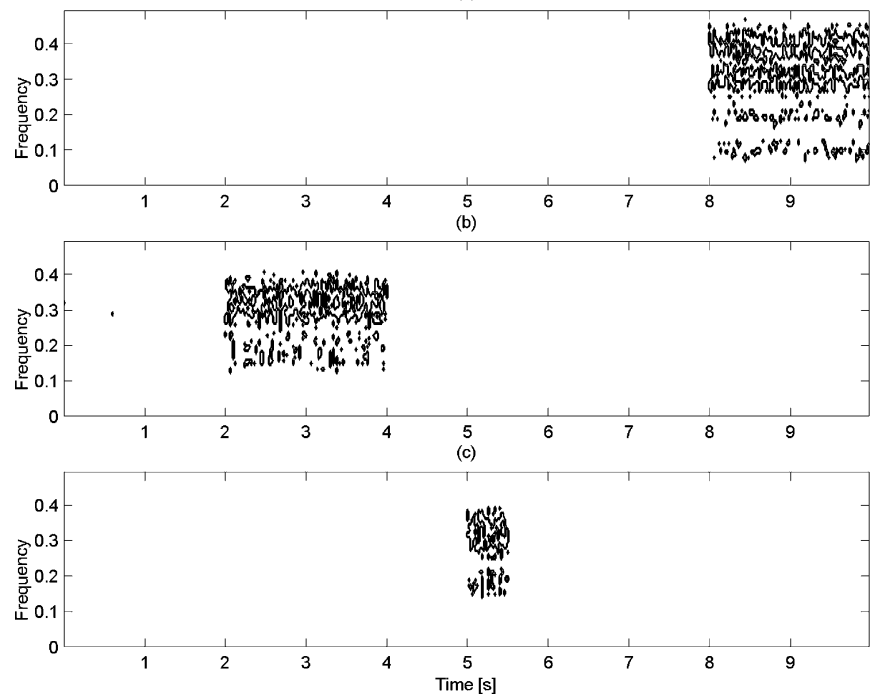

Fig. 11. Separation of three sets at which two sources are present. (a) Source-1 and source- 3 are active. (b) Source- 1 and source- 2 are active. (c) Source- 1 and source- 2 are active.
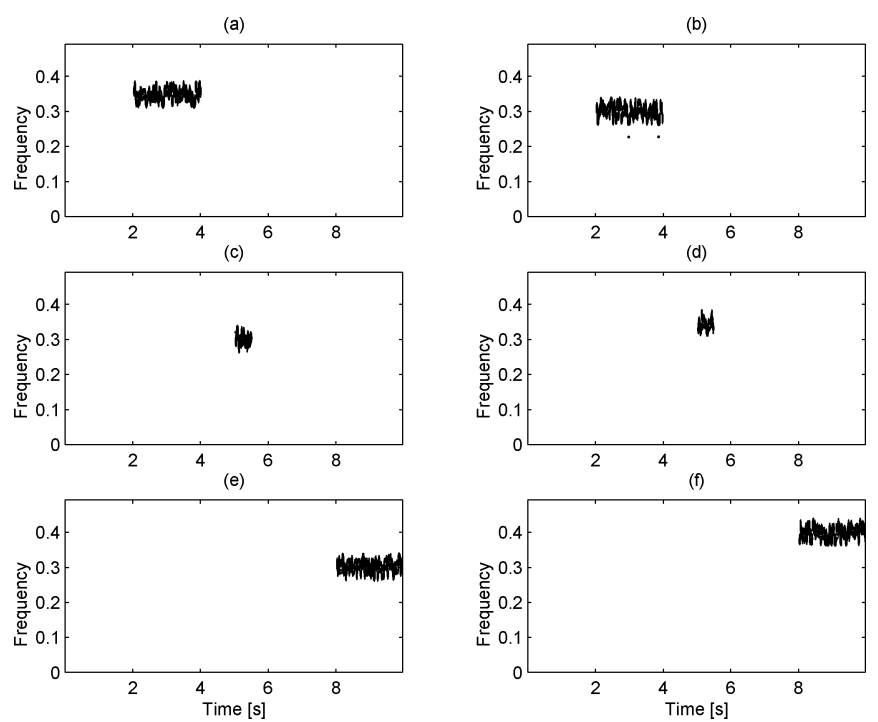

Fig. 12. Time-frequency distribution of the separated signals in the overlap blocks. (a) Estimated signal of source-1. (b) Estimated signal of source-2. (c) Estimated signal of source-1. (d) Estimated signal of source-2. (e) Estimated signal of source-1. (f) Estimated signal of source-3.

The estimation of the block in Fig. 11(c), leads to

$$
\left[\begin{array}{ll}
\hat{\mathbf{a}}_{1} & \hat{\mathbf{a}}_{2}
\end{array}\right]=\left[\begin{array}{cc}
1 & 1 \\
1.0198+0.0399 i & 0.4497-0.9172 i
\end{array}\right] .
$$

Notice that in these two TF blocks, source- 1 and source- 2 are present. The resulting estimates are fairly accurate. For the block in Fig. 11(a), the two estimates of steering vectors are shown.

$$
\left[\begin{array}{ll}
\hat{\mathbf{a}}_{1} & \hat{\mathbf{a}}_{3}
\end{array}\right]=\left[\begin{array}{cc}
1 & 1 \\
0.9992+0.0621 i & -0.9157-0.4087 i
\end{array}\right] .
$$

They are also very close to the actual steering vectors of source- 3 and source- 1 and the overlap also contains these two sources. The TF distributions of the unmixing outputs for each individual block are shown in Fig. 12. The results confirm that successful signal separation is achieved in those overlap blocks
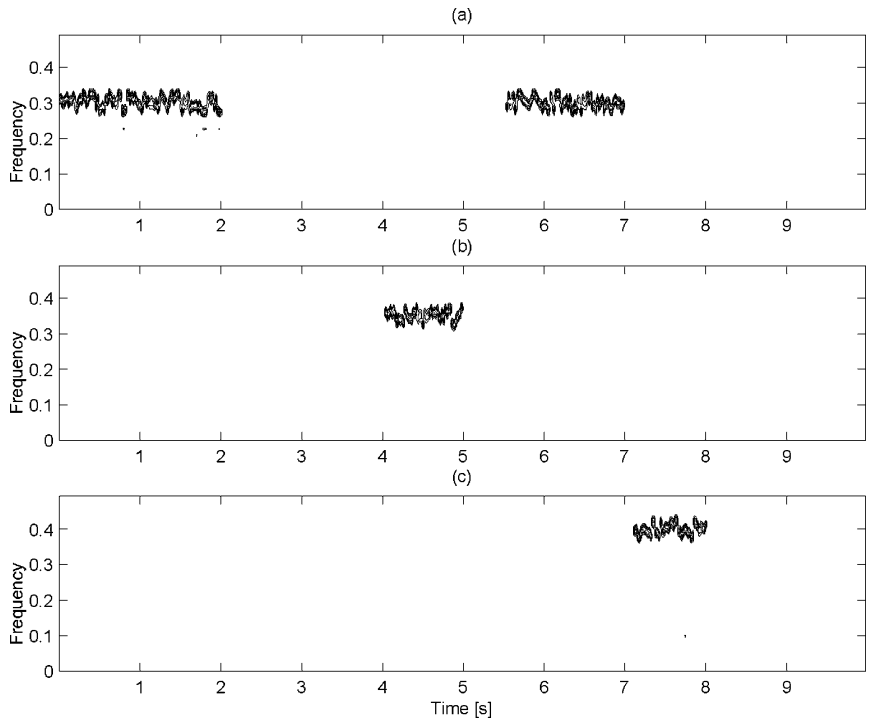

Fig. 13. Separation of the three sets at which only one source is present (a) Source- 1 is active. (b) Source-2 is active. (c) Source- 3 is active.
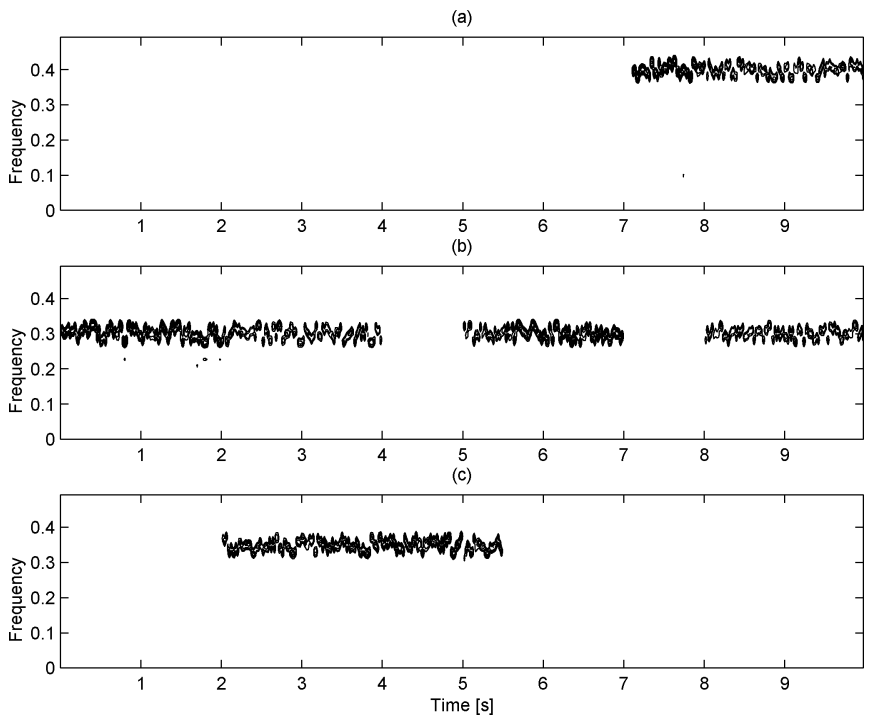

Fig. 14. Successful source separation. (a) Unmixing-1. (b) Unmixing-2. (c) Unmixing-3.

using the JADE algorithm. For the nonoverlap TF blocks, three clusters have been found using the SSCL algorithm. The centers of the clusters are the estimated steering vectors, which are given by (24) at the top of the next page. Signals in those regions are also successfully separated, as shown by the TF distributions of the unmixing output in Fig. 13. With the signal separated in each TF block, the steering vectors in (21)-(24) are gathered together. The DOA is computed and used as the input to the k-means algorithm in order to remove the permutation ambiguity. The unmixed signals in the whole observation period are depicted in the TF domain, as shown in Fig. 14. Successful source separation with fewer sensors is achieved. Notice that the synthesis operation from the TF domain to time domain is no longer required. The signals are well retrieved in the time domain with the proposed method. The unmixing output-2, i.e., source-1, during the interval 2500 to $2600 \mathrm{~s}$ is shown in Fig. 15(b). It is in close similarity to 
$\left[\begin{array}{lll}\hat{\mathbf{a}}_{1} & \hat{\mathbf{a}}_{2} & \hat{\mathbf{a}}_{3}\end{array}\right]=\left[\begin{array}{ccc}1 & 1 & 1 \\ 0.9996-0.0058 i & 0.4818-0.8755 & -0.9199-0.4088 i\end{array}\right]$
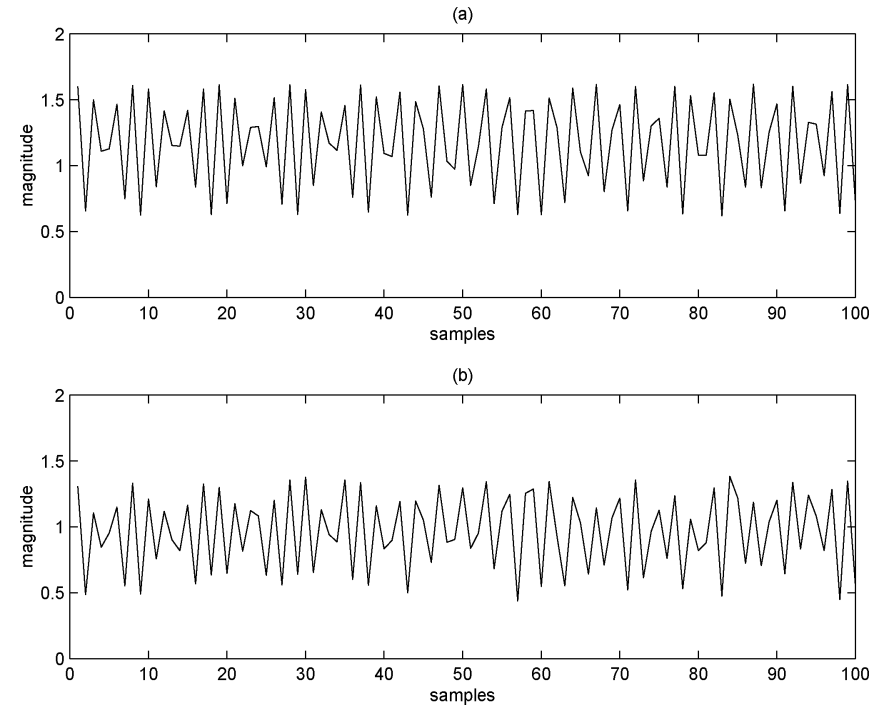

Fig. 15. Time domain signals. (a) Source-1 samples. (b) Unmixing output-2 during the same time interval.

the source-1 signal as shown in Fig. 15(a). Successful source separation is, therefore, achieved.

\section{CONCLUSION}

The problem of underdetermined blind source separation was investigated. The nonstationarity of the input sources was exploited via quadratic TF representation. Under the assumption of TF orthogonality, advanced classification techniques based on Gap statistics and self-splitting competitive learning were introduced to overcome phantom sources. Source separation is, therefore, able to operate in a completely blind fashion without the priori knowledge of the number of sources. The issue when the TF orthogonality assumption is violated was moreover addressed. The TF orthogonality assumption was therefore relaxed to yield a new block-based underdetermined blind source separation algorithm by using the advanced clustering algorithms to partition the TF plane into appropriate blocks. Improvements due to the proposed algorithms were supported by the simulations.

\section{ACKNOWLEDGMENT}

The authors thank the associate editor and three anonymous reviewers for improving the clarity of our paper.

\section{REFERENCES}

[1] J.-F. Cardoso and A. Souloumiac, "Blind beamforming for non Gaussian signals," Inst. Elect. Eng. Proc. F, Radar Signal Process., vol. 140, no. 6, pp. 362-370, Dec. 1993.

[2] A. J. Bell and T. J. Sejnowski, "An information maximization approach to blind separation and blind deconvolution," Neural Comput., vol. 7, no. 6, pp. 1129-1159, Nov. 1995.

[3] S.-I. Amari, A. Cichocki, and H. Yang, "A new learning algorithm for blind signal separation," Adv. Neural Info. Proc. Syst., vol. 8, pp. 757-763, 1996.

[4] T.-W. Lee, Independent Component Analysis: Theory and Applications. Boston, MA: Kluwer Academic, 1998.

[5] S. Haykin, Unsupervised Adaptive Filtering, Volume 1, Blind Source Separation. New York: Wiley, 2000.

[6] A. Hyvärinen, J. Karhunen, and E. Oja, Independent Component Analysis. New York: Wiley, 2001.

[7] A. Cichocki and S.-I. Amari, Adaptive Blind Signal and Image Processing, Learning Algorithm and Applications. New York: Wiley, 2002.

[8] P. Comon, "Independent component analysis: a new concept?," Signal Process., vol. 36, no. 3, pp. 287-314, Apr. 1994.

[9] J.-F. Cardoso, "Blind signal separation: statistical principles," Proc. IEEE, vol. 9, no. 10, pp. 2009-2025, Oct. 1998.

[10] A. Belouchrani and M. G. Amin, "Blind source separation based on time-frequency signal representations," IEEE Trans. Signal Process., vol. 46, no. 11, pp. 2888-2897, 1998.

[11] S. Chen, D. L. Donoho, and M. A. Saunders, "Automic decomposition by basis pursuit," SIAM J. Scientif., vol. 20, no. 1, pp. 33-61, 1998.

[12] T. Lee, M. Lewicki, M. Girolami, and T. Sejnowski, "Blind source separation of more sources than mixtures using overcomplete representations," IEEE Signal Process. Lett., vol. 6, no. 4, pp. 87-90, 1998.

[13] M. S. Lewicki and T. J. Sejnowski, "Learning nonlinear overcomplete representations for efficient coding," Adv. Neural Info. Process. Syst., pp. 815-821, 1998.

[14] - "Learning overcomplete representations," Neural Comput., vol. 12, no. 2, pp. 337-365, 1998.

[15] P. Bofill and M. Zibulevsky, "Underdetermined blind source separation using sparse representation," Signal Process., vol. 81, pp. 2253-2362, 2001.

[16] M. Zibulevsky and B. A. Pearlmutter, "Blind source separation by sparse decomposition in a signal dictionary," Neural Comput., vol. 13, no. 4, pp. 863-882, 2001.

[17] Y. Li, A. Cichocki, and S.-I. Amari, "Sparse composition analysis for blind source separation with less sensors than sources," in Proc. Int. Symp. Independent Component Analysis Blind Signal Separation, Nara, Japan, Apr. 1-4, 2003, pp. 89-94.

[18] K. Waheed and F. M. Salem, "Algebraic overcomplete independent component analysis," in Proc. Int. Symp. Independent Component Analysis Blind Signal Separation, Nara, Japan, Apr. 1-4, 2003, pp. 1077-1080.

[19] L. Vielva, D. Erdogmus, and J. C. Principe, "Underdetermined blind source separation using a probabilistic source sparsity model," presented at the Int. Symp. Independent Component Analysis Blind Signal Separation, 2001.

[20] P. Comon and B. Mourrain, "Decomposition of quantics in sums of powers of linear forms," Signal Process., vol. 52, no. 2, pp. 93-108, 1996.

[21] L. Lathauwer, B. De Moor, J. Vandewalle, and J.-F. Cardoso, "Independent component analysis of largely underdetermined mixture," in Proc. Int. Symp. Independent Component Analysis Blind Signal Separation, Nara, Japan, Apr. 1-4, 2003, pp. 29-33.

[22] B. Chen, A. P. Petropulu, and L. Lathauwer, "Blind identification of convolutive MIMO systems with 3 sources and 2 sensors," EURASIP J. Signal Process., vol. 2002, no. 5, pp. 487-496, 2002. 
[23] I. F. Gorodnitsky and B. D. Rao, "Sparse signal reconstruction from limited data using FOCUSS: A re-weighted minimum norm algorithm," IEEE Trans. Signal Process., vol. 45, no. 3, pp. 600-616, Mar. 1997.

[24] J. F. Murray and K. K. Delgado, "An improved FOCUSS-based learning algorithm for solving blind sparse linear inverse problems," in IEEE Conf. Rec. 35rd Asilomar Conf. Signal, Syst. Comp., vol. 1, Pacific Grove, CA, Nov. 4-7, 2001, pp. 347-351.

[25] K. K. Delgado, J. F. Murray, B. D. Rao, K. Engan, T. W. Lee, and T. J. Sejnowski, "Dictionary learning algorithms for sparse representation," Neural Comput., vol. 15, no. 2, pp. 349-396, 2003.

[26] A. Jourjine, S. Rickard, and O. Yilmaz, "Blind separation of disjoint orthogonal signals: Demixing N sources from 2 mixtures," in Proc. Int. Conf. Acoustics, Speech, Signal Processing (ICASSP), vol. 5, 2000, pp. 2985-2988.

[27] S. Rickard, R. Balan, and J. Rosca, "Real-time time-frequency based blind source separation," in Proc. Int. Conf. Independent Component Analysis Blind Source Separation, San Diego, CA, Dec. 9-12, 2001, pp. 651-656.

[28] — - "Blind source separation based on space-time-frequency diversity," in Proc. Int. Conf. Independent Component Analysis Blind Source Separation, Nara, Japan, Apr. 1-4, 2003, pp. 493-498.

[29] Y. Deville, "Temporal and time-frequency correlation-based blind source separation methods," in Proc. ICA, 2003, pp. 1059-1064.

[30] F. Abrard, Y. Deville, and P. White, "From blind source separation to blind source cancellation in the underdetermined case: A new approach based on time-frequency analysis," in Proc. Int. Conf. Independent Component Analysis Blind Source Separation, San Diego, CA, Dec. 9-12, 2001, pp. 734-739.

[31] L.-T. Nguyen, A. Belouchrain, K. Abed-Meraim, and B. Boashash, "Separating more sources than sensors using time-frequency distribution," in Proc. 6th Int. Symp. Signal Process. Its Applicat., vol. 2, 2001, pp. 583-586.

[32] L. Cohen, "Time-frequency distribution-A review," Proc. IEEE, vol. 77, no. 7, pp. 941-981, 1989.

[33] A. Hardy, "On the number of clusters," Comput. Stat. Data Anal., vol. 23, pp. 83-96, 1996.

[34] T. Hastie, R. Tibshirani, and G. Walther, "Estimating the number of clusters in a dataset via the gap statistic," Stanford Univ., Stanford, CA, Tech. Rep. 208, 2000

[35] Y.-J. Zhang and Z.-Q. Liu, "Self-splitting competitive learning: a new on-line clustering paradigm," IEEE Trans. Neural Netw., vol. 13, no. 2, pp. 369-380, 2002.

[36] F. Hlawatsch and G. F. Boudreaux-Bartels, "Linear and quadratic timefrequency signal representation," IEEE Signal Process. Mag., vol. 9, no. 2, pp. 21-67, 1992

[37] Y. Luo and J. A. Chambers, "Active source selection using Gap statistics for underdetermined blind source separation," Proc. 7th IEEE Int. Symp. Signal Processing Applications (ISSPA), vol. 1, pp. 137-140, 2003.

[38] _ "A modified underdetermined blind source separation method using competitive learning," presented at the ISPA, Rome, Italy, 2003.

[39] _ - "A new block based time-frequency approach for underdetermined blind source separation," presented at the Int. Conf. Acoustics, Speech, Signal Processing (ICASSP), Montreal, QC, Canada, 2004.

[40] F. Auger, P. Flandrin, O. Lemoine, and P. Goncalves. Time-Frequency Toolbox for Matlab. [Online]. Available: http://crttsn.univnantes.fr/ auger/fftb.html

[41] A. Francos and M. Porat, "Analysis and synthesis of multicomponent signals using positive time-frequency distributions," IEEE Trans. Signal Process., vol. 47, no. 2, pp. 493-504, Feb. 1999.

[42] J. B. MacQueen, "Some methods for classification and analysis of multivariate observations," in Proc. 5th Berkeley Symp. Mathemat. Stat. Probab., vol. 1, Berkeley, CA, 1967, pp. 281-297.

[43] J. Hartigan and M. Wong, "A k-means clustering algorithm," Appl. Stat., vol. 28, pp. 100-108, 1979.

[44] H. I. Choi and W. J. Williams, "Improved time-frequency representation of multicomponent signals using exponential kernels," IEEE Trans. Acoust., Speech, Signal Process., vol. 37, no. 6, pp. 862-871, 1989.

[45] I. Takigawa, M. Kudo, and J. Toyama, "Performance analysis of minimum $l_{1}$-norm solutions for underdetermined source separation," IEEE Trans. Signal Process., vol. 52, no. 3, pp. 582-591, Mar. 2004.

[46] F. J. Theis, E. W. Lang, and C. G. Puntonet, "A geometric algorithm for overcomplete linear ICA,” Neurocomputing, vol. 56, pp. 381-398, 2004

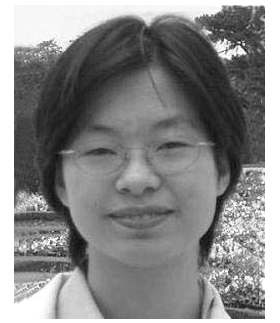

Yuhui Luo (M'03) was born in GuangZhou, China. She received the first class B.Sc. degree in electronic engineering from the University of Central Lancashire, U.K., in 1997, and the M.Sc. and $\mathrm{Ph} . \mathrm{D}$. degrees in digital communications and signal processing from Imperial College of Science, Technology and Medicine, University of London, U.K., in 1998 and 2002, respectively.

From 2002 to 2004, she was a Postdoctoral Research Associate with the Centre for DSP Research, King's College London. She is currently with Samsung Electronics Research Institute, Middlesex, U.K., working in area of radio link control and multimedia integration for UMTS wireless communication systems. Her research interests include statistical signal processing, blind signal processing, and wireless communications.

Dr. Luo received the Best Student Presentation Award for the Fifth International Conference on Mathematics in Signal Processing in 2000. She is a member of the Institute of Electrical Engineers and of the IEEE Signal Processing Society.

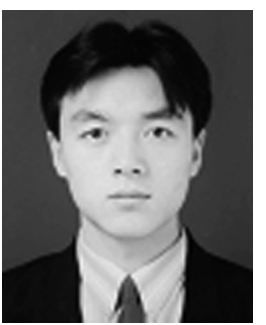

Wenwu Wang (M'03) was born in Anhui, China, in 1974. He received the B.Sc. degree in automatic control in 1997, the M.E. degree in control science and control engineering in 2000, and the Ph.D. degree in navigation guidance and control in 2002, all from Harbin Engineering University, China.

$\mathrm{He}$ joined the Department of Electronic Engineering, King's College London, U.K., as a Postdoctoral Research Associate in May 2002, and transferred to the Cardiff School of Engineering, Cardiff University, U.K., in January 2004. In May 2005, he joined the Tao Group, Ltd., Reading, Berkshire, U.K., where he is currently a DSP engineer working on algorithm design and implementation for multirate signal conversion, echo cancellation, dereverberation and deconvolution, and real-time and embedded system design for audio, multimedia, and graphic engines. His current research interests are in the areas of blind signal processing, audio signal processing, machine learning, and perception.

Dr. Wang is a member of the Institute of Electrical Engineers and a member of the IEEE Signal Processing Society and the IEEE Circuits and Systems Society.

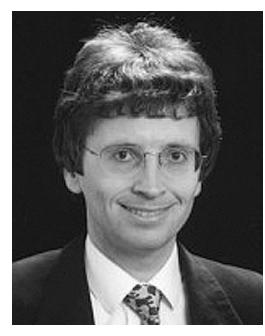

Jonathon A. Chambers (SM'98) was born in Peterborough, U.K., in 1960. He received the B.Sc. (Hons.) degree from the Polytechnic of Central London, U.K., in 1985, together with the Robert Mitchell Medal as the top graduate of the Polytechnic, and the Ph.D. degree from University of London, U.K., in 1990 after study at Peterhouse, Cambridge University, Cambridge, U.K., and Imperial College London.

He served in the Royal Navy as an Artificer Apprentice in Action, Data and Control between 1979 and 1982. He has since held academic and industrial positions with Bath University, Imperial College London, King's College London, and Schlumberger Cambridge Research. In January 2004, he became a Cardiff Professorial Research Fellow with the Cardiff School of Engineering, Cardiff, Wales, U.K. He leads a team of researchers involved in the analysis, design, and evaluation of novel algorithms for digital signal processing with application in acoustics, biomedicine, and wireless communications. His research contributions have been in adaptive and blind signal processing. He has authored and/or coauthored approximately 200 conference and journal publications, and supervised $20 \mathrm{Ph} . \mathrm{D}$. degree graduates.

Dr. Chambers has served as an Associate Editor for the IEEE TRANSACTIONS on Signal Processing and the IEEE TRANSACtions ON Circuits AND Systems. He is a Past Chairman of the IEE Professional Group E5, Signal Processing. He is currently serving as an Associate Editor for the IEEE Signal Processing LetTers, IEEE TRANSACtions on Signal Processing, a Member of IEEE Technical Committee of Signal Processing Theory and Methods, and as the University Liaison on the EURASIP ADCOM Committee. 


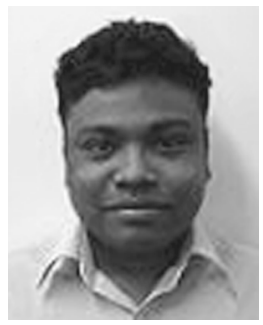

Sangarapillai Lambotharan (M'96) received the B.Eng. Honors First Class degree in electrical and electronic engineering from City University, U.K., in 1994, and the Ph.D. degree in digital signal processing from Imperial College, London, U.K., in 1997.

In August 2005, he joined the Digital Signal Processing Research Group, Cardiff University, U.K., as a Senior Lecturer in signal processing. His previous academic and industrial positions include: Postdoctoral Research Assistant with Imperial College in October 1997 and April 1999, a Visiting Scientist with Cornell University, Ithaca, NY, from August 1996 to December 1996, a Lecturer in Communications and Signal Processing with Brunel University, U.K., from May 1999 to October 1999, a Research Engineer with Motorola, U.K., from November 1999 to January 2002, and a Lecturer in digital signal processing with King's College London from February 2002 to July 2005. His research interests are in the statistical estimation and detection techniques with applications in mobile communications.

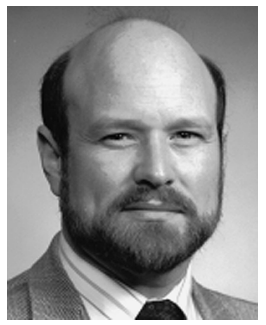

Ian Proudler received a First Class Honors degree in physics from Oxford University, Oxford, U.K., in 1978 and the Ph.D. degree in digital signal processing from the University of Cambridge, Cambridge, U.K., in 1984.

In between his academic degrees, he spent two years in research and development in industry. He remained with the Engineering Department, Cambridge University, until 1986 as a Postdoctoral Researcher working on finite-precision effects in digital filters. He joined QinetiQ (then RSRE, and subsequently, DERA) in 1986 working in the field of adaptive digital signal processing with particular emphasis on least-squares minimization algorithms and architectures. He has published more than 60 research papers and made contributions to four text books.

Dr. Proudler was awarded the 1992 John Benjamin Memorial Prize for his discovery of the QRD-based least-squares lattice algorithm. He received the award again in 2002 for work on the novel KaGE fast least-squares algorithm. $\mathrm{He}$ is the 2002 recipient of the IEE J. J. Thomson Medal "for outstanding work in electronics theory, practice, development or manufacture." He has been the Principal Researcher or a task leader on several advanced research projects. He was elected a QinetiQ Fellow in 1998. He has been on the organizing committee of several international conferences. He was an Honorary Editor for the IEE PROCEEDINGS: RADAR, SONAR AND NAVIGATION for 10 years. 